\title{
The Generalized Linear Sampling Method for limited aperture measurements
}

\author{
Lorenzo Audibert* and Houssem Haddar ${ }^{\dagger}$
}

\begin{abstract}
We extend the so-called Generalized Linear Sampling Method (GLSM) to the case of limited aperture data at a fixed frequency. In this case the factorization of the sampling operator does not obey the symmetry required in the justification of the GLSM introduced in Audibert-Haddar [Inverse Problems, 2014]. We propose a new formulation by adding an extra penalty term that asymptotically correct the non symmetry of the GLSM original penalty term. The analysis of the new formulation is first presented in an abstract framework. We then show how to apply our setting to the scalar problem with far field measurements or near field measurements on a limited aperture. We finally validate the method through some numerical tests in two dimensions and for far field measurements.
\end{abstract}

Key words. Inverse scattering problems, Linear Sampling Method, Generalized Linear Sampling Method, Factorization Method, Qualitative methods

AMS subject classifications. 35R60, 35R30, 65M 32

1. Introduction. This work is concerned with the design of so-called sampling methods $[7,6,8,13,4]$ for inverse scattering problems where one would like to determine the shape of extended targets from fixed frequency multi-static data. More precisely we extend and analyze the recently introduced Generalized Linear Sampling Method [3] (GLSM) to limited aperture data. The GLSM framework developed in [3] provides an exact characterization of the target shape in terms of the so-called far field operator (at fixed frequency and for full aperture). This characterization is based on two factorizations of the far field operator. The first one is used to justify the Linear Sampling Method (LSM) and the second one is at the heart of the Factorization Method(FM). Considering general limited aperture data break the symmetry of the second factorization and prevent the application of the results of [3] or [13] on the FM. The characterization of the GLSM is based on constructing nearby solution to the far field equation as minimizing sequences of a special cost functional. In this cost functional the symmetric factorization is important to ensure that the regularization term has suitable properties. In this article we propose a modification of the regularization term and analyze this modification in order to prove exact characterization even for non symmetric factorization.

The main idea behind our method is that without symmetric factorization it is not possible to control directly the norm of the Herglotz wave that approximately solves the far field equation. However we have access to a term that is close to this quantity and we can bound the error we made, therefore controlling the norm of the associated Herglotz wave. Due to this splitting the control is coarser and therefore it reflects the fact that this situation is less favorable for imaging. The fact that the regularization involves compact operators or the case of noisy operators are covered using the idea already proposed in [3]. However the interesting property of strong convergence of the minimizing sequence of the cost functional demonstrated

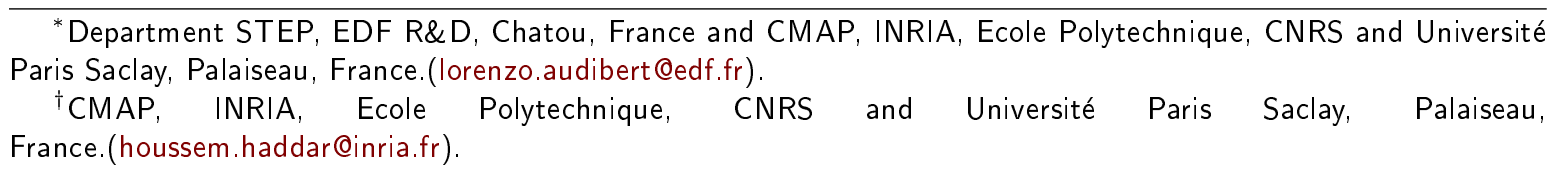


in [2] could not be simply extended. The second main contribution of this article is to add a regularization term to lower the hypothesis of [2] on the regularization term. This new results extend the validity of the results of [2] and enable an extension to non symmetric factorization. In order to introduce those ideas we choose to present the case of scalar inverse scattering from inhomogeneous inclusions for limited aperture far field measurements. We also indicate how the method can be easily extended to near field data.

On the numerical side we introduce a second order method to minimize the cost functional, this method prove to be more efficient than the one use in [3]. The superiority of our indicator function is demonstrated for symmetric factorization. The theory does not say how to choose the regularization parameter for symmetric factorization, the method does not seem to be very sensitive and an heuristic choice give good result. For non symmetric factorization this choice is by far more important and we propose three heuristics to set this parameter.

The article is organized as follows.In Section 2 a model problem is introduced to motivate the GLSM for non symmetric factorization. Theoretical extension for the symmetric factorization is given in section 3.1 and the case of non-symmetric factorization is treated in section 3.2. Section 4 provides an example of application by completely treating the model problem introduce in section 2. Section 5 show how nearfield data easily fit into the theory developed in Section 3. The last section (Section 6) is devoted to numerical algorithms issued from section 4 along with validating numerical results and discussion on the difference between symmetric and non-symmetric cases.

2. A model problem for limited aperture data. We choose to present our method for the simple model of inverse time harmonic scattering problem from inhomogeneous targets. For a wave number $k>0$, the total field solve the following scalar wave equation:

$$
\Delta u+k^{2} n u=0 \text { in } \mathbb{R}^{d}
$$

with $d=2$ or 3 and with $n \in L^{\infty}\left(\mathbb{R}^{d}\right)$ denoting the refractive index such that the support of $n-1$ is included inside $\bar{D}$ with $D$ a bounded domain with Lipschitz boundary and connected complement and such that $\Im(n) \geq 0$.

We consider the cases where the total field is generated by incident plane waves, $u^{i}(\theta, x):=$ $e^{i k x \cdot \theta}$ with $x \in \mathbb{R}^{d}$ and $\theta \in \Gamma_{s}\left(\Gamma_{s} \subset \mathbb{S}^{d-1}\right.$ the unit sphere $)$ and we denote by $u^{s}$ the scattered field defined by

$$
u^{s}(\theta, \cdot)=u-u^{i}(\theta, \cdot) \quad \text { in } \mathbb{R}^{d},
$$

which is assumed to be satisfying the Sommerfeld radiation condition,

$$
\lim _{r \rightarrow \infty} \int_{|x|=r}\left|\frac{\partial u^{s}}{\partial r}-i k u^{s}\right|^{2} d s=0 .
$$

The data for the inverse problem is formed by noisy measurements of the so called far field pattern $u^{\infty}(\theta, \hat{x})$ defined by

$$
u^{s}(\theta, x)=\frac{e^{i k|x|}}{|x|^{(d-1) / 2}}\left(u^{\infty}(\theta, \hat{x})+O(1 /|x|)\right)
$$


as $|x| \rightarrow \infty$ for all $(\theta, \hat{x}) \in \Gamma_{s} \times \Gamma_{m}$, where $\Gamma_{m}$ is a subset of $\mathbb{S}^{d-1}$ possibly different from $\Gamma_{s}$. The goal is to be able to reconstruct $D$ from these measurements (without knowing $n$ ). We introduce the far field operator $F: L^{2}\left(\Gamma_{s}\right) \rightarrow L^{2}\left(\Gamma_{m}\right)$, defined by

$$
F g(\hat{x}):=\int_{\Gamma_{s}} u^{\infty}(\theta, \hat{x}) g(\theta) d s(\theta), \hat{x} \in \Gamma_{m}
$$

68 Let us define, for $\psi \in L^{2}(D)$, the unique function $w \in H_{\mathrm{loc}}^{1}\left(\mathbb{R}^{d}\right)$ satisfying

$$
\left\{\begin{array}{l}
\Delta w+n k^{2} w=-k^{2}(n-1) \psi \text { in } \mathbb{R}^{d} \\
\lim _{r \rightarrow \infty} \int_{|x|=r}\left|\frac{\partial w}{\partial r}-i k w\right|^{2} d s=0 .
\end{array}\right.
$$

By linearity of the forward scattering problem, $F g$ is nothing but the far field pattern of $w$ solution of (1) with $\psi=v_{g}$ in $D$, where

$$
v_{g}(x):=\int_{\Gamma_{s}} e^{i k x \cdot \theta} g(\theta) d s(\theta), g \in L^{2}\left(\Gamma_{s}\right), x \in \mathbb{R}^{d} .
$$

70 Now consider the (compact) operator $H_{s}: L^{2}\left(\Gamma_{s}\right) \rightarrow L^{2}(D)$ defined by

$$
H_{s} g:=\left.v_{g}\right|_{D},
$$

72 and the (compact) operator $G_{m}: \overline{\mathcal{R}\left(H_{s}\right)} \subset L^{2}(D) \rightarrow L^{2}\left(\Gamma_{m}\right)$ defined by

$$
G_{m} \psi:=\left.w^{\infty}\right|_{\Gamma_{m}}
$$

where $w^{\infty}$ is the far field of $w \in H_{\mathrm{loc}}^{1}\left(\mathbb{R}^{d}\right)$ solution of (1) and where $\overline{\mathcal{R}\left(H_{s}\right)}$ denotes the closure of the range of $H_{s}$ in $L^{2}(D)$. Then clearly

$$
F=G_{m} H_{s}
$$

one simply has $G_{m}=H_{m}^{*} T \psi$, where $H_{m}^{*}: L^{2}(D) \rightarrow L^{2}\left(\Gamma_{m}\right)$ is the adjoint of the operator $H_{m}$ (defined similarly to $H_{s}$ but with $\Gamma_{s}$ replaced by $\Gamma_{m}$ ) and whose expression is given by

$$
H_{m}^{*} \varphi(\hat{x}):=\int_{D} e^{-i k y \cdot \hat{x}} \varphi(y) d y, \varphi \in L^{2}(D), \hat{x} \in \Gamma_{m},
$$

78 and where the operator $T: L^{2}(D) \rightarrow L^{2}(D)$ is defined by

$$
T \psi:=-k^{2}(1-n)(\psi+w),
$$


with $w \in H_{\mathrm{loc}}^{1}\left(\mathbb{R}^{d}\right)$ being the solution of (1). Finally we end up with

$$
F=H_{m}^{*} T H_{s} .
$$

This factorization is called "non symmetric" in the cases $H_{m} \neq H_{s}$ which correpond to $\Gamma_{s} \neq \Gamma_{m}$. The GLSM as formulated in [3] applies to the "symmetric" cases, i.e. $\Gamma_{s}=\Gamma_{m}$. Physically the latter correspond with sources and receivers on symmetric opposite sides of the target (as shown in Figure 1).
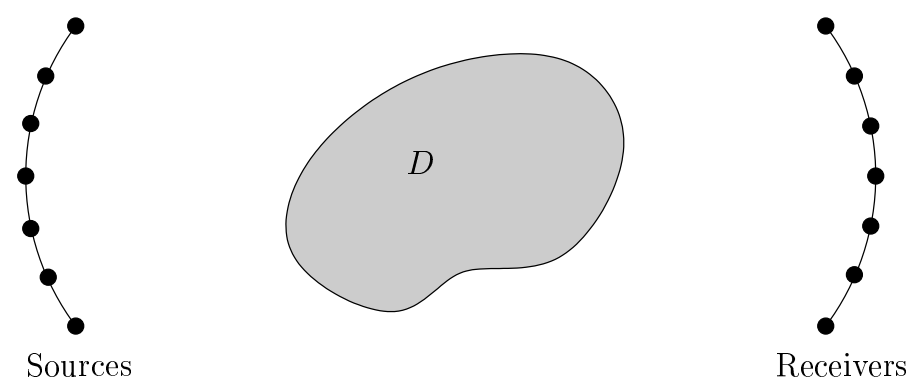

Figure 1. Sources-receivers configurations that correspond with symmetric factorizations of the far field operator.

Our focus in the following is to extend the GLSM to non symmetric factorizations of the measurement operator.

3. Theoretical foundation of the GLSM for limited aperture. In this section we shall give the theoretical foundation of the extension of the GLSM to non symmetric factorizations. We will adopt an abstract framework that can be applied to other settings than the one presented in the previous section (See for instance Section 5 where the case of near field data is considered). As pointed above, the "symmetry" in the factorization of the far field operator is of primary importance in the GLSM framework of [3] where the following cost functional (for noise-free data) was introduced:

$$
J_{\alpha}(\phi ; g):=\alpha|\langle B g, g\rangle|+\alpha^{1-\eta}|\langle F g-\phi, g\rangle|+\|F g-\phi\|^{2}
$$

with $B$ being an operator constructed from $F$ and that has a "symmetric" factorization. The latter seems to be hard to ensure in general when $F$ itself has not a "symmetric" factorization. In some special cases this can be done as for heterogeneous backgrounds [10] or special settings of the near field data [5]. However, in the case of limited aperture presented above with $\Gamma_{m} \neq \Gamma_{s}$, this type of construction seems to be impossible to achieve. This is why we shall consider in the following only the case $B=F$.

As has been pointed out in [2], for the case $B=F$, one cannot guarantee in general the strong convergence of Herglotz waves associated with the minimizing sequences of $J_{\alpha}(\phi ; g)$ (when the sampling point is inside $D$ ). Since this convergence is an important property for some imaging algorithms (as in [2] for the case of differential measurements), we shall first modify the setting of GLSM so that one obtain this convergence result even in the case $B=F$. The idea is to add an extra (carefully chosen) penalty term that is inspired from difficulties encountered in establishing the over mentioned convergence result in the classical setting of GLSM. 


\subsection{A new formulation of the GLSM for symmetric factorizations.}

3.1.1. Analysis of the noise free case. We denote by $X$ and $Y$ two (complex) reflexive Banach spaces with duals $X^{*}$ and $Y^{*}$ respectively and shall denote by $\langle$,$\rangle a duality product$ that refers to $\left\langle X^{*}, X\right\rangle$ or $\left\langle Y^{*}, Y\right\rangle$ duality. We consider the linear operator $F: X \rightarrow X^{*}$. Moreover we shall assume that the following factorization holds

$$
F=H^{*} T H
$$

where the operators $H: X \rightarrow Y$ and $T: Y \rightarrow Y^{*}$ are bounded. We denote by $G: \overline{\mathcal{R}(H)} \subset$ $Y \rightarrow X^{*}$ the linear operator $H^{*} T$ restricted to $\overline{\mathcal{R}(H)}$.

Let $\alpha>0$ be a given parameter and $\phi \in X^{*}$. The new GLSM (for noise free measurements) is based on considering minimizing sequences of the functional $J_{\alpha}(\phi ; \cdot): X \rightarrow \mathbb{R}$

$$
J_{\alpha}(\phi ; g):=\alpha|\langle F g, g\rangle|+\alpha^{1-\eta}|\langle F g-\phi, g\rangle|+\|F g-\phi\|^{2} \quad \forall g \in X,
$$

where $\eta \in] 0,1]$ is a fixed parameter. Following [3], we first observe that

$$
j_{\alpha}(\phi):=\inf _{g \in X} J_{\alpha}(\phi ; g) \rightarrow 0 \text { as } \alpha \rightarrow 0 .
$$

for all $\phi \in X^{*}$ if one assumes that $F$ has dense range. Indeed in this case, for a given $\varepsilon>0$ there exists $g_{\varepsilon}$ such that $\left\|F g_{\varepsilon}-\phi\right\|<\frac{\varepsilon}{2}$. Then one can choose $\alpha_{0}(\varepsilon)$ such for all $\alpha \leq \alpha_{0}(\varepsilon)$, $\alpha\left|\left\langle F g_{\varepsilon}, g_{\varepsilon}\right\rangle\right|+\alpha^{1-\eta}|\langle F g-\phi, g\rangle|<\frac{\varepsilon}{2}$ so that $j_{\alpha}(\phi)<\varepsilon$, which proves (8). One then can prove the following characterization of the range of $G$ in terms of $F$ : .

Theorem 1. We assume that $H$ is compact, $G$ is injective and $F$ is injective with dense range. We also assume that $T$ satisfies the coercivity property

$$
|\langle T h, h\rangle|>\mu\|h\|^{2} \quad \forall h \in \mathcal{R}(H),
$$

where $\mu>0$ is a constant independent of $h$. Consider for $\alpha>0$ and $\phi \in X^{*}, g_{\alpha} \in X$ such that

$$
J_{\alpha}\left(\phi ; g_{\alpha}\right) \leq j_{\alpha}(\phi)+p(\alpha)
$$

where $\frac{p(\alpha)}{\alpha}$ is bounded with respect to $\alpha$. Then

$$
\phi \in \mathcal{R}(G) \quad \text { iff } \quad \lim _{\alpha \rightarrow 0}\left|\left\langle F g_{\alpha}, g_{\alpha}\right\rangle\right|<\infty .
$$

In the case $\phi=G \varphi$, the sequence $H g_{\alpha}$ converges strongly to $\varphi$ in $Y$ as $\alpha$ goes to zero.

Proof. Assume that $\phi \in \mathcal{R}(G)$ and let $\varphi \in \overline{\mathcal{R}(H)}$ such that $G \varphi=\phi$. For $\alpha>0$ one can choose $g_{0} \in X$ such that $\left\|H g_{0}-\varphi\right\|^{2}<\alpha^{2}$. Then by continuity of $G,\left\|F g_{0}-\phi\right\|^{2}<\|G\|^{2} \alpha^{2}$. On the other hand the continuity of $T$ implies

$$
\left|\left\langle F g_{0}, g_{0}\right\rangle\right|=\left|\left\langle T H g_{0}, H g_{0}\right\rangle\right| \leq\|T\|\left\|H g_{0}\right\|^{2}<2\|T\|\left(\alpha^{2}+\|\varphi\|^{2}\right)
$$

and

$$
\left|\left\langle F g_{0}-\phi, g_{0}\right\rangle\right|=\left|\left\langle T\left(H g_{0}-\varphi\right), H g_{0}\right\rangle\right| \leq\|T\|\left\|H g_{0}-\varphi\right\|\left\|H g_{0}\right\|<2\|T\| \alpha(\alpha+\|\varphi\|) .
$$


From the definitions of $j_{\alpha}(\phi)$ and $g_{\alpha}$ we have

$$
\alpha\left|\left\langle F g_{0}, g_{0}\right\rangle\right|+\alpha^{1-\eta}\left|\left\langle F g_{0}-\phi, g_{0}\right\rangle\right|+\left\|F g_{0}-\phi\right\|^{2}>j_{\alpha}(\phi)>J_{\alpha}\left(\phi, g_{\alpha}\right)-p(\alpha) .
$$

We then deduce from the definition of $J_{\alpha}$, the fact that $\left.\left.\eta \in\right] 0,1\right]$ and previous inequalities

(11) $\alpha\left|\left\langle F g_{\alpha}, g_{\alpha}\right\rangle\right| \leq J_{\alpha}\left(\phi, g_{\alpha}\right) \leq p(\alpha)+2 \alpha\|T\|\left(\alpha^{2}+\|\varphi\|^{2}\right)+\alpha^{2}\|G\|^{2}+2\|T\| \alpha^{2-\eta}(\alpha+\|\varphi\|)$.

Therefore $\limsup _{\alpha \rightarrow 0}\left|\left\langle F g_{\alpha}, g_{\alpha}\right\rangle\right|<\infty$. The coercivity property of $T$ implies that $\left\|H g_{\alpha}\right\|^{2}$ is bounded. From (8) and (10) and the injectivity of $G$ we infer that the only possible weak limit of (any subsequence of) $H g_{\alpha}$ is $\varphi$. Thus the whole sequence $H g_{\alpha}$ weakly converges to $\varphi$ in $Y$. On the other hand we have that:

$$
\begin{aligned}
\left\|H g_{\alpha}-\varphi\right\|^{2} & \leq\left|\left\langle T\left(H g_{\alpha}-\varphi\right), H g_{\alpha}-\varphi\right\rangle\right| \\
& \leq\left|\left\langle T\left(H g_{\alpha}-\varphi\right), H g_{\alpha}\right\rangle\right|+\left|\left\langle T\left(H g_{\alpha}-\varphi\right), \varphi\right\rangle\right| \\
& \leq\left|\left\langle F g_{\alpha}-\phi, g_{\alpha}\right\rangle\right|+\left|\left\langle T\left(H g_{\alpha}-\varphi\right), \varphi\right\rangle\right|
\end{aligned}
$$

The last term goes to zero due to the weak convergence of $H g_{\alpha}$. The first term goes to zero since the second inequality in (11) implies in particular that $\left|\left\langle F g_{\alpha}-\phi, g_{\alpha}\right\rangle\right| \leq \alpha^{\eta}$. Therefore we conclude that $H g_{\alpha}$ strongly converges to $\varphi$ and consequently

$$
\lim _{\alpha \rightarrow 0}\left|\left\langle F g_{\alpha}, g_{\alpha}\right\rangle\right|=|\langle T \varphi, \varphi\rangle| .
$$

We now consider the case $\phi \notin \mathcal{R}(G)$. Assume that $\liminf _{\alpha \rightarrow 0}\left|\left\langle F g_{\alpha}, g_{\alpha}\right\rangle\right|<\infty$. Then, (for some extracted subsequence $\left.g_{\alpha}\right)\left|\left\langle F g_{\alpha}, g_{\alpha}\right\rangle\right|<A$ for some constant $A$ independent of $\alpha \rightarrow 0$. The coercivity of $T$ implies that $\left\|H g_{\alpha}\right\|$ is also bounded and therefore one can assume that, up to an extracted subsequence, $H g_{\alpha}$ weakly converges to some $\varphi \in \overline{\mathcal{R}(H)}$. Since $G$ is compact, we obtain that $G H g_{\alpha}$ strongly converges to $G \varphi$ as $\alpha \rightarrow 0$. On the other hand, (8) and the definition of $J_{\alpha}\left(\phi, g_{\alpha}\right)$ imply that $\left\|F g_{\alpha}-\phi\right\| \leq J_{\alpha}\left(\phi, g_{\alpha}\right) \leq j_{\alpha}(\phi)+C \alpha \rightarrow 0$ as $\alpha \rightarrow 0$. Since $F g_{\alpha}=G H g_{\alpha}$ we obtain that $G \varphi=\phi$ which is a contradiction. We then conclude that if $\phi \notin \mathcal{R}(G)$ then $\lim _{\alpha \rightarrow 0}\left|\left\langle F g_{\alpha}, g_{\alpha}\right\rangle\right|=\infty$.

Remark 1. The extension proposed in Theorem 1 requires indeed less assumptions to ensure strong convergence than the one proposed in [2] for the case of symmetric factorizations. However the result from [2] is still interesting for practical applications (when applicable) since it uses a convex cost functional which is easier to minimize numerically.

3.1.2. Analysis for the case of noisy measurements. Let $F^{\delta}: X \rightarrow X^{*}$ be the operator associated with noisy far field measurements such that

$$
\left\|F^{\delta}-F\right\| \leq \delta
$$

for some $\delta>0$. We assume that the operators $F^{\delta}$ and $F$ are compact. Again let $\left.\left.\eta \in\right] 0,1\right]$ be a fixed parameter. We define for $\alpha>0$ and $\phi \in X^{*}$ the regularized functional

$$
J_{\alpha}^{\delta}(\phi ; g):=\alpha\left|\left\langle F^{\delta} g, g\right\rangle\right|+\alpha^{1-\eta}\left|\left\langle F^{\delta} g-\phi, g\right\rangle\right|+\alpha^{1-\eta} \delta\|g\|^{2}+\left\|F^{\delta} g-\phi\right\|^{2}
$$


154 we get

Consequently, following the same arguments as for the second inequality in (11), we arrive at

$$
\alpha\left(\left|\left\langle F^{\delta} g_{\alpha}^{\delta}, g_{\alpha}^{\delta}\right\rangle\right|+\alpha^{-\eta} \delta\left\|g_{\alpha}^{\delta}\right\|^{2}\right) \leq J_{\alpha}^{\delta}\left(\phi ; g_{\alpha}^{\delta}\right) \leq C \alpha,
$$

for sufficiently small $\alpha$ with $C$ a constant independent from $\alpha$. This proves $\limsup _{\alpha \rightarrow 0} \limsup _{\delta \rightarrow 0}\left(\left|\left\langle F^{\delta} g_{\alpha}^{\delta}, g_{\alpha}^{\delta}\right\rangle\right|+\alpha^{-\eta} \delta \| F^{\delta}\right.$ $\infty$. We also have, as a consequence of the inequalities above, that

$$
\delta\left\|g_{\alpha}^{\delta}\right\|^{2} \leq C \alpha^{\eta}
$$


which proves $\limsup _{\alpha \rightarrow 0} \lim _{\delta \rightarrow 0} \delta\left\|g_{\alpha}^{\delta}\right\|^{2}=0$. We also have

$$
\left|\left\langle F^{\delta} g_{\alpha}^{\delta}-\phi, g_{\alpha}^{\delta}\right\rangle\right| \leq C \alpha^{\eta}
$$

which proves, with the estimate on $\delta\left\|g_{\alpha}^{\delta}\right\|^{2}$ given above, that

$$
\lim _{\alpha \rightarrow 0} \limsup _{\delta \rightarrow 0}\left|\left\langle F g_{\alpha}^{\delta}-\phi, g_{\alpha}^{\delta}\right\rangle\right|=0 .
$$

We then get that $\lim _{\alpha \rightarrow 0} \limsup _{\delta \rightarrow 0}\left|\left\langle F^{\delta} g_{\alpha}^{\delta}, g_{\alpha}^{\delta}\right\rangle\right|<\infty$ and can conclude as in the proof of Theorem 1 that $H g_{\alpha}^{\delta(\alpha)}$ converges strongly to $\varphi$ as $\alpha$ goes to zero for $\delta(\alpha)$ sufficiently small. This also proves that $\lim _{\alpha \rightarrow 0} \limsup _{\delta \rightarrow 0}\left(\left|\left\langle F^{\delta} g_{\alpha}^{\delta}, g_{\alpha}^{\delta}\right\rangle\right|+\delta \alpha^{-\eta}\left\|g_{\alpha}^{\delta}\right\|^{2}\right)<\infty$.

Now assume that $\phi \notin \mathcal{R}(G)$ and $\liminf _{\alpha \rightarrow 0} \liminf _{\delta \rightarrow 0}\left(\left|\left\langle F^{\delta} g_{\alpha}^{\delta}, g_{\alpha}^{\delta}\right\rangle\right|+\alpha^{-\eta} \delta\left\|g_{\alpha}^{\delta}\right\|^{2}\right)$ is finite. The coercivity of $T$ and $\alpha<1$ implies that

$$
\mu\left\|H g_{\alpha(\delta)}^{\delta}\right\|^{2} \leq\left|\left\langle F g_{\alpha}^{\delta}, g_{\alpha}^{\delta}\right\rangle\right| \leq\left|\left\langle F^{\delta} g_{\alpha}^{\delta}, g_{\alpha}^{\delta}\right\rangle\right|+\alpha^{-\eta} \delta\left\|g_{\alpha}^{\delta}\right\|^{2}
$$

Therefore $\liminf _{\alpha \rightarrow 0} \liminf _{\delta \rightarrow 0}\left\|H g_{\alpha}^{\delta}\right\|^{2}$ is also finite. This means the existence of a subsequence $\left(\alpha^{\prime}, \delta\left(\alpha^{\prime}\right)\right)$ such that $\alpha^{\prime} \rightarrow 0$ and $\delta\left(\alpha^{\prime}\right) \rightarrow 0$ as $\alpha^{\prime} \rightarrow 0$ and $\left\|H g_{\alpha^{\prime}}^{\delta\left(\alpha^{\prime}\right)}\right\|^{2}$ is bounded independently from $\alpha^{\prime}$. One can also choose $\delta\left(\alpha^{\prime}\right)$ such that $\delta\left(\alpha^{\prime}\right) \leq \alpha^{\prime 1-\eta}$. On the other hand Equation (14) indicates that one can choose this subsequence such that $J_{\alpha^{\prime}}^{\delta\left(\alpha^{\prime}\right)}\left(g_{\alpha^{\prime}}^{\delta\left(\alpha^{\prime}\right)}\right) \rightarrow 0$ as $\alpha^{\prime} \rightarrow 0$ and therefore $\left\|F^{\delta} g_{\alpha^{\prime}}^{\delta\left(\alpha^{\prime}\right)}-\phi\right\| \rightarrow 0$ as $\alpha^{\prime} \rightarrow 0$ and $\alpha^{\prime 1-\eta} \delta\left(\alpha^{\prime}\right)\left\|g_{\alpha^{\prime}}^{\delta\left(\alpha^{\prime}\right)}\right\|^{2} \rightarrow 0$ as $\alpha^{\prime} \rightarrow 0$. By a triangular inequality and $\delta\left(\alpha^{\prime}\right) \leq \alpha^{1-\eta}$ we then deduce that $\left\|F g_{\alpha^{\prime}}^{\delta\left(\alpha^{\prime}\right)}-\phi\right\| \rightarrow 0$ as $\alpha^{\prime} \rightarrow 0$. The compactness of $G$ implies that a subsequence of $G H g_{\alpha^{\prime}}^{\delta\left(\alpha^{\prime}\right)}$ converges for some $G \varphi$ in $X^{*}$. The uniqueness of the limit implies that $G \varphi=\phi$, which is a contradiction.

3.2. The GLSM for non symmetric factorizations. In this section we shall extend GLSM formalism presented in the previous section to the case of non symmetric factorisations. The general framework is given by the following assumptions. We shall denote by $X_{1}, X_{2}$ and $Y$ three (complex) reflexive Banach spaces with duals $X_{1}^{*}, X_{2}^{*}$ and $Y^{*}$ respectively and shall denote by $\langle$,$\rangle a duality product that refers to \left\langle X_{1}^{*}, X_{1}\right\rangle,\left\langle X_{2}^{*}, X_{2}\right\rangle$ or $\left\langle Y^{*}, Y\right\rangle$ duality. We also set $X:=X_{1} \times X_{2}$.

We consider a linear operator $F: X_{2} \rightarrow X_{1}^{*}$ that is assumed to be bounded and has the following factorization

$$
F=U^{*} T V
$$

where the operators $V: X_{2} \rightarrow Y, T: Y \rightarrow Y^{*}$ and $U: X_{1} \rightarrow Y$ are bounded. We set $G: \overline{\mathcal{R}_{Y}(V)} \subset Y \rightarrow X_{1}^{*}$ the restriction of $U^{*} T$ to $\overline{\mathcal{R}_{Y}(V)}$ where $\overline{\mathcal{R}_{Y}(V)}$ is the closure of the 
range of $V$ in $Y$. We shall assume in addition the existence of a space $\hat{Y}$ such that $U$ and $V$ can be extended to bounded operators $V: X_{2} \rightarrow \hat{Y}$ and $U: X_{1} \rightarrow \hat{Y}$ such that

$$
\left\|V g_{2}+U g_{1}\right\|_{Y} \leq\left\|V g_{2}+U g_{1}\right\|_{\hat{Y}}, \quad \forall\left(g_{1}, g_{2}\right) \in X .
$$

We finally assume that

$$
\overline{\mathcal{R}_{Y}(V)}=\overline{\mathcal{R}_{Y}(U)} \text { and } \overline{\mathcal{R}_{\hat{Y}}(V)}=\overline{\mathcal{R}_{\hat{Y}}(U)} .
$$

A typical example is the case of limited aperture presented above with $X_{2}=L^{2}\left(\Gamma_{s}\right)$, $X_{1}=L^{2}\left(\Gamma_{m}\right), Y=L^{2}(D)$ and $\hat{Y}=L^{2}(\Sigma)$ with $\Sigma$ being any domain such that $D \subset \Sigma$. The domain $\Sigma$ is assumed to be known a priori (which can coincide with the whole probed domain) and therefore the operators $V: X_{2} \rightarrow \hat{Y}$ and $U: X_{1} \rightarrow \hat{Y}$ are also known a priori. In the case of limited aperture presented above these operators are defined by

$$
V g(x)=\int_{\Gamma_{s}} e^{i k x \cdot \theta} g(\theta) d s(\theta) \text { and } U g(x)=\int_{\Gamma_{m}} e^{i k x \cdot \theta} g(\theta) d s(\theta), \quad x \in \Sigma .
$$

3.2.1. Analysis of the noise free case. Let $\alpha>0$ be a given parameter and $\phi \in X_{1}^{*}$. We redefine the functional $J_{\alpha}$ as $J_{\alpha}(\phi ; \cdot): X=X_{1} \times X_{2} \rightarrow \mathbb{R}$

$$
J_{\alpha}(\phi ; g):=\alpha\left|\left\langle F g_{2}, g_{1}\right\rangle\right|+\alpha^{1-\eta}\left\|V g_{2}-U g_{1}\right\|_{\hat{Y}}^{2}+\alpha^{1-\eta}\left|\left\langle F g_{2}-\phi, g_{1}\right\rangle\right|+\left\|F g_{2}-\phi\right\|^{2}
$$

for all $g=\left(g_{1}, g_{2}\right) \in X$ where $\left.\eta \in\right] 0,1[$ is again a fixed parameter. We also set

$$
j_{\alpha}(\phi):=\inf _{g \in X} J_{\alpha}(\phi ; g) .
$$

Indeed the role of the extra term $\left\|V g_{2}-U g_{1}\right\|$ is to formally ensure $V g_{2} \simeq U g_{1}$ which cannot be done exactly since the ranges of the operators $V$ and $U$ are different in general. We then observe that the penalty term is of the form

$$
\left\langle F g_{2}, g_{1}\right\rangle \mid=\left\langle T V g_{2}, U g_{1}\right\rangle \simeq\left\langle T V g_{2}, V g_{2}\right\rangle
$$

and therefore formally behaves as in the case of symmetric factorizations. The goal of the following analysis is to show that this is indeed asymptotically the case as $\alpha \rightarrow 0$. We first prove that with the additional penalty term, the inf still goes to 0 as $\alpha \rightarrow 0$ which guarantee that we can construct nearby solutions of the $F g \simeq \phi$.

Lemma 3. Assume that $F$ has dense range. Then for all $\phi \in X_{1}^{*}, j_{\alpha}(\phi) \rightarrow 0$ as $\alpha \rightarrow 0$.

Proof. Since $F$ has dense range, for a given $\varepsilon>0$ there exists $g_{2}^{\varepsilon}$ such that

$$
\left\|F g_{2}^{\varepsilon}-\phi\right\| \leq \varepsilon / 3 .
$$

Using (18) and (17) we can choose $g_{1}^{\varepsilon}$ such that:

$$
\left\|V g_{2}^{\varepsilon}-U g_{1}^{\varepsilon}\right\|_{Y}^{2}<\left\|V g_{2}^{\varepsilon}-U g_{1}^{\varepsilon}\right\|_{\hat{Y}}^{2}<\varepsilon / 3
$$


Together with inequalities (21) and (22) the latter inequality implies

$$
j_{\alpha}(\phi) \leq J_{\alpha}\left(\phi ; g^{\varepsilon}\right) \leq \varepsilon
$$

for sufficiently small $\alpha$ where $g^{\varepsilon}:=\left(g_{1}^{\varepsilon}, g_{2}^{\varepsilon}\right)$.

We now can state and prove the main theorem of this section that provides a characterization of the range of $G$ in terms of $F$ and $U$ and $V$ as operators with values in $\hat{Y}$.

Theorem 4. We assume that $G: \overline{\mathcal{R}_{Y}(V)} \subset Y \rightarrow X_{1}^{*}$ is injective and that $F$ has dense range. We also assume that $T$ satisfies the coercivity property

$$
|\langle T \varphi, \varphi\rangle|>\mu\|\varphi\|^{2} \quad \forall \varphi \in \overline{\mathcal{R}(U)}=\overline{\mathcal{R}(V)},
$$

where $\mu>0$ is a constant independent of $\varphi$. Let $p(\alpha)$ be a given function such that $\frac{p(\alpha)}{\alpha}=O(1)$ and consider for $\alpha>0$ and $\phi \in X_{1}^{*}, g^{\alpha}=\left(g_{1}^{\alpha}, g_{2}^{\alpha}\right) \in X$ such that

$$
J_{\alpha}\left(\phi ; g^{\alpha}\right) \leq j_{\alpha}(\phi)+p(\alpha) .
$$

211 Then we have the following:

212• $\phi \in \mathcal{R}(G)$ implies $\limsup _{\alpha \rightarrow 0}\left(\left|\left\langle F g_{2}^{\alpha}, g_{1}^{\alpha}\right\rangle\right|+\alpha^{-\eta}\left\|V g_{2}^{\alpha}-U g_{1}^{\alpha}\right\|_{\hat{Y}}^{2}\right)<\infty$.

$\phi \notin \mathcal{R}(G)$ implies $\lim _{\alpha \rightarrow 0}^{\alpha \rightarrow 0}\left(\left|\left\langle F g_{2}^{\alpha}, g_{1}^{\alpha}\right\rangle\right|+\alpha^{-\eta}\left\|V g_{2}^{\alpha}-U g_{1}^{\alpha}\right\|_{\hat{Y}}^{2}\right)=\infty$

214 In the case $\phi=G \varphi$, the two sequences $V g_{2}^{\alpha}$ and $U g_{1}^{\alpha}$ strongly converge to $\varphi$ in $Y$.

Proof. The proof follows roughly the same steps and ideas as the proof for the case of symmetric factorizations. We start with the case $\phi \in \mathcal{R}(G)$. We consider $\varphi \in \overline{\mathcal{R}_{Y}(V)}$ such that $G \varphi=\phi$ and $h_{2}^{\alpha} \in X_{2}$ such that $\left\|V h_{2}^{\alpha}-\varphi\right\|_{Y}^{2} \leq \alpha^{2}$. According to (18) and (17), there exists $h_{1}^{\alpha} \in X_{1}$ such that:

$$
\begin{aligned}
\left|\left\langle F h_{2}^{\alpha}, h_{1}^{\alpha}\right\rangle\right| & =\left|\left\langle T V h_{2}^{\alpha}, U h_{1}^{\alpha}\right\rangle\right| \\
& \leq\left|\left\langle T V h_{2}^{\alpha}, V h_{2}^{\alpha}\right\rangle\right|+\left|\left\langle T V h_{2}^{\alpha}, U h_{1}^{\alpha}-V h_{2}^{\alpha}\right\rangle\right| \\
& \leq\|T\|\left\|V h_{2}^{\alpha}\right\|_{Y}^{2}+\|T\|\left\|V h_{2}^{\alpha}\right\|_{Y} \sqrt{\alpha^{\eta}}
\end{aligned}
$$

and

$$
\begin{aligned}
\left|\left\langle F h_{2}^{\alpha}-\phi, h_{1}^{\alpha}\right\rangle\right|=\left|\left\langle T\left(V h_{2}^{\alpha}-\varphi\right), U h_{1}^{\alpha}\right\rangle\right| & \leq\|T\|\left\|V h_{2}^{\alpha}-\varphi\right\|\left\|U h_{1}^{\alpha}\right\| \\
& <2\|T\| \alpha\left(\alpha+\|\varphi\|+\sqrt{\alpha^{\eta}}\right) .
\end{aligned}
$$

The two previous inequalities and the definitions $g^{\alpha}$ and $j_{\alpha}(\phi)$ lead to

$$
\alpha\left(\left|\left\langle F g_{2}^{\alpha}, g_{1}^{\alpha}\right\rangle\right|+\alpha^{-\eta}\left\|V g_{2}^{\alpha}-U g_{1}^{\alpha}\right\|_{\hat{Y}}^{2}+\alpha^{-\eta}\left|\left\langle F h_{2}^{\alpha}-\phi, h_{1}^{\alpha}\right\rangle\right|\right) \leq j_{\alpha}(\phi)+p(\alpha) \leq C \alpha,
$$




$$
\left|\left\langle F g_{2}^{\alpha}-\phi, g_{1}^{\alpha}\right\rangle\right| \leq C \alpha^{\eta} .
$$

We shall prove now the convergence of $V g_{2}^{\alpha}$ strongly converges to $\varphi$ in $Y$ where $G \varphi=\phi$. The coercivity of $T$ implies

$\mu\left\|V g_{2}^{\alpha}\right\|_{Y}^{2} \leq\left|\left\langle T V g_{2}^{\alpha}, V g_{2}^{\alpha}\right\rangle\right| \leq\left|\left\langle T V g_{2}^{\alpha}, V g_{2}^{\alpha}\right\rangle+\left\langle T V g_{2}^{\alpha}, U g_{1}^{\alpha}-V g_{2}^{\alpha}\right\rangle\right|+\left|\left\langle T V g_{2}^{\alpha}, U g_{1}^{\alpha}-V g_{2}^{\alpha}\right\rangle\right|$

On the one hand

$$
\left|\left\langle T V g_{2}^{\alpha}, V g_{2}^{\alpha}\right\rangle+\left\langle T V g_{2}^{\alpha}, U g_{1}^{\alpha}-V g_{2}^{\alpha}\right\rangle\right|=\left|\left\langle F g_{2}^{\alpha}, g_{1}^{\alpha}\right\rangle\right| \leq C
$$

and on the other hand

$$
\left|\left\langle T V g_{2}^{\alpha}, U g_{1}^{\alpha}-V g_{2}^{\alpha}\right\rangle\right| \leq\|T\|\left\|V g_{2}^{\alpha}\right\|_{Y}\left\|V g_{2}^{\alpha}-U g_{1}^{\alpha}\right\|_{\hat{Y}} \leq\|T\| C \alpha^{\eta}\left\|V g_{2}^{\alpha}\right\|_{Y}
$$

These inequalities show that $\left\|V g_{2}^{\alpha}\right\|_{Y}$ is bounded. Second, from Lemma 3 and (25) and the injectivity of $G$ we infer that the only possible weak limit of (any subsequence of) $V g_{2}^{\alpha}$ in $Y$ is $\varphi$. Thus the whole sequence $V g_{2}^{\alpha}$ weakly converges to $\varphi$ in $Y$. Following the idea of proof of Theorem 1 we use the formula:

$$
\left|\left\langle T\left(V g_{2}^{\alpha}-\varphi\right), V g_{2}^{\alpha}-\varphi\right\rangle\right| \leq\left|\left\langle T\left(V g_{2}^{\alpha}-\varphi\right), \varphi\right\rangle\right|+\underbrace{\left|\left\langle T\left(V g_{2}^{\alpha}-\varphi\right), V g_{2}^{\alpha}-U g_{1}^{\alpha}\right\rangle\right|}_{\leq\|T\|\left(\left\|V g_{2}^{\alpha}\right\|+\|\varphi\|\right)\left\|V g_{2}^{\alpha}-U g_{1}^{\alpha}\right\|_{Y}}+\left|\left\langle F g_{2}^{\alpha}-\phi, g_{1}^{\alpha}\right\rangle\right|
$$

The first term on the right hand side goes to zero thanks to the weak convergence, the second term goes to zero thanks to (28) and the third term goes to zero thanks to (29). The coercivity property of $T$ implies that $V g_{2}^{\alpha}$ converges strongly to $\varphi$ in $Y$. The strong convergence of $U g_{1}^{\alpha}$ to $\varphi$ in $Y$ is a direct consequence of (28).

We now consider the case $\phi \notin \mathcal{R}(G)$ and assume that $\liminf _{\alpha \rightarrow 0}\left|\left\langle F g_{2}^{\alpha}, g_{1}^{\alpha}\right\rangle\right|+\alpha^{-\eta}\left\|V g_{2}^{\alpha}-U g_{1}^{\alpha}\right\|_{\hat{Y}}^{2}$ $\infty$. Then, (for some extracted subsequence $g^{\alpha}$ ) $\left|\left\langle F g_{2}^{\alpha}, g_{1}^{\alpha}\right\rangle\right|+\alpha^{-\eta}\left\|V g_{2}^{\alpha}-U g_{1}^{\alpha}\right\|_{\hat{Y}}^{2} \leq A$ for some $A$ independent of $\alpha$ as $\alpha$ goes to 0 . Using the same reasoning as in the first part of the theorem this implies that $\left\|V g_{2}^{\alpha}\right\|_{Y}$ is bounded. We then obtain a contradiction exactly in the same way as in the proof of the second part of Theorem 1.

3.2.2. Analysis of the case of perturbed operators. We now consider the case of noisy data and/or non exact models. The noise in the data is modelled with an operator $F^{\delta}$ such that

$$
\left\|F^{\delta}-F\right\| \leq \delta
$$

for some $\delta>0$. We can also assume error in the "model" by considering perturbed operators $U^{\delta}, V^{\delta}$

$$
\left\|U^{\delta}-U\right\| \leq \delta \text { and }\left\|V^{\delta}-V\right\| \leq \delta .
$$




$$
\begin{array}{cl}
J_{\alpha}^{\delta}(\phi ; g):=\alpha & \left|\left\langle F^{\delta} g_{2}, g_{1}\right\rangle\right|+\delta \alpha^{1-\eta}\left(\left\|g_{1}\right\|_{X_{1}}^{2}+\left\|g_{2}\right\|_{X_{2}}^{2}\right)+\alpha^{1-\eta}\left|\left\langle F^{\delta} g_{2}-\phi, g_{1}\right\rangle\right| \\
& +\alpha^{1-\eta}\left\|V^{\delta} g_{2}-U^{\delta} g_{1}\right\|_{\hat{Y}}^{2}+\left\|F^{\delta} g_{2}-\phi\right\|_{X_{1}^{*}}^{2}
\end{array}
$$

for $g=\left(g_{1}, g_{2}\right) \in X$. We can also treat the case of noisy incorrect knowledge of $\phi$ by assume that one would consider $\phi^{\delta} \in X$ such that

$$
\left\|\phi^{\delta}-\phi\right\| \leq \delta
$$

$$
\begin{aligned}
n(\delta, \alpha, g):= & \delta\left(\alpha+\alpha^{1-\eta}\right)\left(\left\|g_{1}\right\|_{X_{1}}^{2}+\left\|g_{2}\right\|_{X_{2}}^{2}\right) \\
& +\delta^{2}\left(\left\|g_{2}\right\|_{X_{2}}^{2}+\alpha^{1-\eta}\left(\left\|g_{1}\right\|_{X_{1}}^{2}+\left\|g_{2}\right\|_{X_{2}}^{2}\right)+1\right) .
\end{aligned}
$$

Lemma 5. For for all $\alpha, \delta>0$ the functional $J_{\alpha}^{\delta}\left(\phi^{\delta} ; \cdot\right)$ has a minimizer $g^{\alpha, \delta}$. Assume in addition that $F$ has dense range. Then we have

$$
\lim _{\alpha \rightarrow 0} \lim _{\delta \rightarrow 0} J_{\alpha}^{\delta}\left(\phi^{\delta} ; g^{\alpha, \delta}\right)=0
$$

Proof. The existence of a minimizer is clear: for a fixed $\alpha>0, \delta>0$ and $\phi^{\delta}$, any minimizing sequence $g^{n}$ of $J_{\alpha}^{\delta}\left(\phi^{\delta} ; \cdot\right)$ is bounded and therefore there exists a weakly convergent subsequence to some $g^{\alpha, \delta}$. The lower semi-continuity of the norm with respect to the weak convergence and the compactness property of the operators then imply:

$$
J_{\alpha}^{\delta}\left(\phi^{\delta} ; g^{\alpha, \delta}\right) \leq \liminf _{n \rightarrow+\infty} J_{\alpha}^{\delta}\left(\phi^{\delta} ; g^{n}\right) \leq \inf _{g} J_{\alpha}^{\delta}\left(\phi^{\delta} ; g\right)
$$

which proves that $g^{\alpha, \delta}$ is a minimizer of $J_{\alpha}^{\delta}(\phi ; \cdot)$. Let $\epsilon>0$ be given. We consider $g^{\varepsilon}$ as introduced in the proof of Lemma 3 and choose $\delta$ sufficiently small $\left(\delta \leq \delta_{0}(\alpha, \epsilon)\right.$ such that

$$
n\left(\delta, \alpha, g^{\varepsilon}\right) \leq \varepsilon
$$

We then deduce from (31) and the definition of $g^{\alpha, \delta}$ that

$$
J_{\alpha}^{\delta}\left(\phi^{\delta} ; g^{\alpha, \delta}\right) \leq J_{\alpha}\left(\phi ; g^{\varepsilon}\right)+\varepsilon
$$

and conclude as in Lemma 3 that

$$
J_{\alpha}^{\delta}\left(\phi ; g^{\alpha, \delta}\right) \leq 2 \varepsilon
$$

for sufficiently small $\alpha$, which proves the second claim of the lemma. 
We now can prove the following asymptotic characterization of the range of $G$ (as $\delta 0$ ). In order to shorten the notaion we define

$$
R(g, \alpha, \delta):=\left|\left\langle F^{\delta} g_{2}, g_{1}\right\rangle\right|+\delta \alpha^{-\eta}\left(\left\|g_{1}\right\|_{X_{1}}^{2}+\left\|g_{2}\right\|_{X_{2}}^{2}\right)+\alpha^{-\eta}\left\|V^{\delta} g_{2}-U^{\delta} g_{1}\right\|_{\hat{Y}}^{2} .
$$

244

245

247• $\phi \in \mathcal{R}(G)$ implies lim sup lim sup $R($

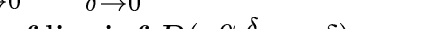

248• $\phi \notin \mathcal{R}(G)$ implies $\liminf _{\alpha \rightarrow 0} \liminf _{\delta \rightarrow 0} R\left(g^{\alpha, \delta}, \alpha, \delta\right)=\infty$

249 Moreover, if $G \varphi=\phi$, then we also have

$$
\limsup _{\alpha \rightarrow 0} \limsup _{\delta \rightarrow 0} \delta\left(\left\|g_{1}^{\alpha, \delta}\right\|_{X_{1}}^{2}+\left\|g_{2}^{\alpha, \delta}\right\|_{X_{2}}^{2}\right)=0
$$

251

and there exists $\delta_{0}(\alpha)$ such that for all $\delta(\alpha) \leq \delta_{0}(\alpha), V g_{2}^{\alpha, \delta(\alpha)}$ and $U g_{1}^{\alpha, \delta(\alpha)}$ converge strongly to $\varphi$ in $Y$ as $\alpha$ goes to zero.

Proof. Consider first the case $\phi \in \mathcal{R}(G)$. We shall make use of same function $h^{\alpha}=\left(h_{1}^{\alpha}, h_{2}^{\alpha}\right)$ as in the first part of the proof of Theorem 4 (that only depends on $\alpha$ ). If we choose $\delta(\alpha)$ such that:

$$
n\left(\delta(\alpha), \alpha, h^{\alpha}\right) \leq \alpha
$$

(where $n$ is defined in (32)) then we get (as in first part of the proof of Theorem 4)

$$
J_{\alpha}^{\delta}\left(\phi ; g^{\alpha, \delta}\right) \leq C \alpha+\alpha
$$

$$
R\left(g^{\alpha, \delta}, \alpha, \delta\right) \leq C
$$

which proves the first assertion of the theorem. We also get, as a consequence of the inequalities above, that

$$
\delta\left(\left\|g_{1}^{\alpha, \delta}\right\|_{X_{1}}^{2}+\left\|g_{2}^{\alpha, \delta}\right\|_{X_{2}}^{2}\right) \leq C \alpha^{\eta}
$$

255 which proves

$$
\lim _{\alpha \rightarrow 0} \limsup _{\delta \rightarrow 0} \delta\left(\left\|g_{1}^{\alpha, \delta}\right\|_{X_{1}}^{2}+\left\|g_{2}^{\alpha, \delta}\right\|_{X_{2}}^{2}\right)=0
$$

257 For the same reasons, since $\eta>0$ we have

$$
\lim _{\alpha \rightarrow 0} \limsup _{\delta \rightarrow 0}\left\|V^{\delta} g_{2}^{\alpha, \delta}-U^{\delta} g_{1}^{\alpha, \delta}\right\|_{\hat{Y}}^{2}=0 .
$$

Now choose $\delta_{0}(\alpha)$ small enough such that, $\limsup _{\alpha \rightarrow 0} n\left(\delta_{0}(\alpha), \alpha, h^{\alpha}\right)=0$, consider $\delta(\alpha) \leq \delta_{0}(\alpha)$ and denote by $\tilde{g}^{\alpha}:=g^{\alpha, \delta(\alpha)}$. Then, from (34) and (36) we clearly obtain that the quantity 
$\left\langle T V^{\delta(\alpha)} \tilde{g}_{2}^{\alpha}, V^{\delta(\alpha)} \tilde{g}_{2}^{\alpha}\right\rangle$ is bounded. To conclude as in the proof of Theorem 4 that $V \tilde{g}_{2}^{\alpha}$ and $U \tilde{g}_{1}^{\alpha}$ converge strongly to $\varphi$ in $Y$ as $\alpha$ goes to zero, one just need to remark that

$$
\left\|V \tilde{g}_{2}^{\alpha}-U \tilde{g}_{1}^{\alpha}\right\|_{\hat{Y}}^{2} \leq\left\|V^{\delta(\alpha)} \tilde{g}_{2}^{\alpha}-U^{\delta(\alpha)} \tilde{g}_{1}^{\alpha}\right\|_{\hat{Y}}^{2}+\delta(\alpha)^{2}\left\|\tilde{g}_{1}^{\alpha}\right\|_{X_{1}}^{2}+\delta(\alpha)^{2}\left\|\tilde{g}_{2}^{\alpha}\right\|_{X_{2}}^{2} \rightarrow 0
$$

as $\alpha \rightarrow 0$ and

$$
\left|\left\langle F \tilde{g}_{2}^{\alpha}-\phi, \tilde{g}_{1}^{\alpha}\right\rangle\right| \leq\left|\left\langle F^{\delta(\alpha)} \tilde{g}_{2}^{\alpha}-\phi, \tilde{g}_{1}^{\alpha}\right\rangle\right|+\delta\left(\left\|\tilde{g}_{1}^{\alpha}\right\|_{X_{1}}^{2}+\left\|\tilde{g}_{2}^{\alpha}\right\|_{X_{2}}^{2}\right) \rightarrow 0 .
$$

We recall that it is known [15] that if $n-1$ positive definite or negative definite in a neighborhood of $\partial D$, Hypothesis 1 is verified for all $k \in \mathbb{R}$ except a countable set without finite accumulation point.

Defining

$$
\phi_{z}(\hat{x}):=e^{-i k \hat{x} \cdot z} \text { for } \hat{x} \in \Gamma_{m}
$$

278 we have:

Consider now the case $\phi \notin \mathcal{R}(G)$ and assume that $\liminf _{\alpha \rightarrow 0} \liminf _{\delta \rightarrow 0} R\left(g^{\alpha, \delta}, \alpha, \delta\right)$ is finite. Then, from

$$
\left|\left\langle F g_{2}^{\alpha, \delta}, g_{1}^{\alpha, \delta}\right\rangle\right| \leq\left|\left\langle F^{\delta} g_{2}^{\alpha, \delta}, g_{1}^{\alpha, \delta}\right\rangle\right|+\frac{\delta}{2}\left\|g_{1}^{\alpha, \delta}\right\|_{X_{1}}^{2}+\frac{\delta}{2}\left\|g_{2}^{\alpha, \delta}\right\|_{X_{2}}^{2}
$$

we deduce that $\left|\left\langle F g_{2}^{\alpha, \delta}, g_{1}^{\alpha, \delta}\right\rangle\right|$ is bounded for some subsequence $\delta(\alpha)$. One also get that $\left\|V g_{2}^{\alpha, \delta}-U g_{1}^{\alpha, \delta}\right\|_{\hat{Y}}^{2}$ is bounded for the same sequence $\delta(\alpha)$ meaning that, similarly to the second part of the proof of Theorem 4, the sequence $\left\|V g_{2}^{\alpha, \delta(\alpha)}\right\|_{Y}$ is bounded as $\alpha \rightarrow 0$. We then can obtain a contradiction exactly the same way as in the proof of Theorem 4.

4. Application to inverse scattering. The purpose of this section is to apply the result of section 3 to limited aperture data (described in section 2). This will be possible if $D \subset \Sigma$ where $\Sigma$ is some bounded known domain. We then define $\hat{Y}$ from section 3 to be $L^{2}(\Sigma)$ and set $V=H_{s}$ and $U=H_{m}$.

The basis of the GLSM is the characterization of the obstacle $D$ in term of the range of $G_{m}$. This characterization is based on the solvability of the following interior transmission problem for $u, v \in L^{2}(D)$ such that $u-v \in H^{2}(D)$,

$$
\left\{\begin{array}{l}
\Delta u+k^{2} n u=0 \quad \text { in } D \\
\Delta v+k^{2} v=0 \quad \text { in } D \\
(u-v)=f \quad \text { on } \partial D \\
\frac{\partial}{\partial \nu}(u-v)=g \quad \text { on } \partial D
\end{array}\right.
$$

Hypothesis 1 . We assume that $k^{2} \in \mathbb{R}_{+}$is such that for all $f \in H^{\frac{3}{2}}(\partial D)$ and $g \in H^{\frac{1}{2}}(\partial D)$ problem (38) has a unique solution in $(u, v) \in L^{2}(D) \times L^{2}(D)$ and $u-v \in H^{2}(D)$. 
Theorem 7. Under Hypothesis 1, $\phi_{z} \in \mathcal{R}\left(G_{m}\right)$ (for $G_{m}$ defined in (3)) if and only if $z \in D$. Lemma 8. $\overline{\mathcal{R}\left(H_{s}\right)}=\left\{v \in L^{2}(D)\right.$ s.t. $\Delta v+k^{2} v=0$ in $\left.D\right\}=\overline{\mathcal{R}\left(H_{m}\right)}$

The proof of this theorem is rather straightforward using the result of Lemma 8 (see [13]) and the fact that $\phi_{z}$ is the far field of $\Phi(\cdot, z)$, the fundamental solution of the Helmholtz equation satisfying the Sommerfeld radiation condition.

The central additional theorem needed in order to apply the theory developed in Section 3 is the following coercivity property of the operator $T$. This theorem holds true under the following assumptions:

Hypothesis 2. We assume that $n \in L^{\infty}\left(\mathbb{R}^{d}\right)$ with $\Im(n) \geq 0$ and there exist constants $n_{0}, \alpha>$ 0 such that $1-\Re(n)+\alpha \Im(n) \geq 0$ for a.e. $x$ in a neighborhood of $\partial D$ or $\Re(n)-1+\alpha \Im(n) \geq 0$ for a.e. $x$ in a neighborhood of $\partial D$.

The following lemma has been proved in [1].

Lemma 9. Assume that Hypothesis 2 holds and that $k^{2}$ is not a transmission eigenvalue. Then the operator $T$ satisfies the coercivity property (9).

Let $C>0$ be a given constant (independent of $\alpha$ ) and consider $\alpha>0$ and $z \in \mathbb{R}^{d}$, $g^{z, \alpha}=\left(g_{1}^{z, \alpha}, g_{2}^{z, \alpha}\right) \in L^{2}\left(\Gamma_{m}\right) \times L^{2}\left(\Gamma_{s}\right)$ such that :

$$
\begin{aligned}
& J_{\alpha}\left(\phi_{z}, g^{z, \alpha}\right)=\alpha\left|\left\langle F g_{2}^{z, \alpha}, g_{1}^{z, \alpha}\right\rangle\right|+\alpha^{1-\eta}\left\|H_{s} g_{2}^{z, \alpha}-H_{m} g_{1}^{z, \alpha}\right\|_{L^{2}(\Sigma)}^{2} \\
& +\alpha^{1-\eta}\left|\left\langle F g_{2}^{z, \alpha}-\phi_{z}, g_{1}^{z, \alpha}\right\rangle\right|+\left\|F g_{2}^{z, \alpha}-\phi_{z}\right\|^{2} \\
& \leq j_{\alpha}\left(\phi_{z}\right)+C \alpha,
\end{aligned}
$$

where $\eta \in] 0,1[$ and

$$
j_{\alpha}\left(\phi_{z}\right)=\inf _{g \in L^{2}\left(\Gamma_{m}\right) \times L^{2}\left(\Gamma_{s}\right)} J_{\alpha}\left(\phi_{z}, g\right) .
$$

Combining the results of Theorems 4 and 7 we obtain the following theorem:

Theorem 10. Assume that Hypotheses 1 and 2 hold. Then $z \in D$ if and only if $\limsup _{\alpha \rightarrow 0}\left|\left\langle F g_{2}^{z, \alpha}, g_{1}^{z, \alpha}\right\rangle\right|+$ $\alpha^{-\eta}\left\|H_{s} g_{2}^{z, \alpha}-H_{m} g_{1}^{z, \alpha}\right\|_{L^{2}(\Sigma)}^{2}<\infty$.

Moreover, if $z \in D$ then the sequence of Herglotz wave functions associated to $g^{z, \alpha}$ converges strongly to the solution $v$ of $(38)$ with $(f, g)=\left(\Phi_{z}, \frac{\partial \Phi_{z}}{\partial \nu}\right)$ as $\alpha$ goes to zero.

For applications, it is important to rather use the criterion provided in Theorem 6 . Consider $F^{\delta}: L^{2}\left(\Gamma_{s}\right) \rightarrow L^{2}\left(\Gamma_{m}\right)$ a compact operator such that:

$$
\left\|F^{\delta}-F\right\| \leq \delta
$$

Then consider for $\alpha>0$ and $\phi \in L^{2}\left(\Gamma_{m}\right)$ the functional $J_{\alpha}^{\delta}(\phi, \cdot): L^{2}\left(\Gamma_{s}\right) \times L^{2}\left(\Gamma_{m}\right) \rightarrow \mathbb{R}$,

$$
\begin{aligned}
& J_{\alpha}^{\delta}\left(\phi_{z}, g\right)=\alpha\left|\left\langle F^{\delta} g_{2}, g_{1}\right\rangle\right|+\alpha^{1-\eta}\left\|H_{s} g_{2}-H_{m} g_{1}\right\|_{L^{2}(\Sigma)}^{2}+\alpha^{1-\eta} \delta\|g\|^{2} \\
& +\alpha^{1-\eta}\left|\left\langle F^{\delta} g_{2}-\phi_{z}, g_{1}\right\rangle\right|+\left\|F^{\delta} g_{2}-\phi_{z}\right\|^{2}
\end{aligned}
$$

where $\eta \in] 0,1[$. Then as a direct consequences of Theorem 6 we obtain the following characterization of $D$, 
Theorem 11. Assume that the hypothesis of Theorem 10 hold true. For $z \in \mathbb{R}^{d}$ denote by $g^{z, \alpha, \delta}$ the minimizer of $J_{\alpha}^{\delta}(\phi, \cdot)$ over $L^{2}\left(\Gamma_{s}\right) \times L^{2}\left(\Gamma_{m}\right)$. Then $z \in D$ if and only if

$$
\underset{\alpha \rightarrow 0}{\limsup } \limsup _{\delta \rightarrow 0}\left|\left\langle F^{\delta} g_{2}^{z, \alpha, \delta}, g_{1}^{z, \alpha, \delta}\right\rangle\right|+\alpha^{-\eta}\left\|H_{s} g_{2}^{z, \alpha, \delta}-H_{m} g_{1}^{z, \alpha, \delta}\right\|_{L^{2}(\Sigma)}^{2}+\alpha^{-\eta} \delta\left\|g^{z, \alpha, \delta}\right\|^{2}<\infty \text {. }
$$

If $z \in D$, there exists $\delta_{0}(\alpha)$ such that for all $\delta(\alpha) \leq \delta_{0}(\alpha), H g^{z, \alpha, \delta(\alpha)}$ converges strongly to the solution $v$ of $(38)$ with $(f, g)=\left(\Phi_{z}, \frac{\partial \Phi_{z}}{\partial \nu}\right)$ as $\alpha$ goes to zero.

5. Extension to near field data. We concentrated in the previous sections on incident plane waves and far field measurement and raise the problem of "non symmetric factorization" in the case of limited apertures. We here show how the theory of Section 3 can be applied to other configurations of non symmetric factorization. This is the case for instance of near field data that we shall present in this section.

The total field is generated by point sources and the scattered field is recorded on a surface of $\mathbb{R}^{d}$ (usually where the point source lies). If we denote by $\partial \Omega$ the surface where the sources lie, we consider an incident field $u^{i}(y, x):=\Phi(y, x)$ with $x \in \mathbb{R}^{d}$ and $y \in \partial \Omega$. We introduce $N: H^{-\frac{1}{2}}(\partial \Omega) \rightarrow H^{\frac{1}{2}}(\partial \Omega)$ defined by

$$
N g:=\int_{\partial \Omega} u^{s}(y, x) g(y) d s(y), g \in H^{-\frac{1}{2}}(\partial \Omega), x \in H^{\frac{1}{2}}(\partial \Omega),
$$

where $u^{s}(y, \cdot=w)$ solution of (1) with an incident field $\psi=u^{i}(y, \cdot)$. We introduce the compact operator $S: H^{-\frac{1}{2}}(\partial \Omega) \rightarrow L^{2}(D)$ (which plays the role of $H_{s}$ ) defined by

$$
S g:=\int_{\partial \Omega} \Phi(y, x) g(y) d s(y), g \in H^{-\frac{1}{2}}(\partial \Omega), x \in D
$$

and the (compact) operator $G: \overline{\mathcal{R}(S)} \subset L^{2}(D) \rightarrow H^{\frac{1}{2}}(\partial \Omega)$ defined by

$$
G \psi:=\left.w\right|_{\partial \Omega},
$$

where $\overline{\mathcal{R}(S)}$ denotes the closure of the range of $S$ in $L^{2}(D)$ and $w$ is defined as in (1). Then clearly

$$
N=G S .
$$

In the case under consideration, since the scattered field has the following expression :

$$
w(x)=-\int_{D} \Phi(y, x)(1-n) k^{2}(\psi(y)+w(y)) d y,
$$

one simply has $G=\bar{S}^{*} T \psi$ where $\bar{S}^{*}: L^{2}(D) \rightarrow L^{2}(\partial \Omega)$ is the conjugate of the adjoint of $S$ given by:

$$
\bar{S}^{*} \varphi(x)=\int_{D} \Phi(y, x) \varphi(y) d y, x \in \Gamma,
$$

and $T$ is defined by (4). Finally we get

$$
N=\bar{S}^{*} T S
$$

As for the limited aperture case this factorization is "non symmetric". 
5.1. Point sources and point measurements on the same surface. The case where the point sources and the measurements are on the same surface can be solved without relying on the theory developed in Section 3.2. At the cost of computing an operator $C$ (introduced in the following) such that :

$$
B=C F=H^{*} T H,
$$

one can rely on the theory of Section 3.1 or on the theory proposed in [3]. In [13] an inf-criterion is proposed to tackle the case of near field full aperture, through the use of the corresponding far field operator. We refer to [12] for similar ideas using near field measurement. We propose to adapt this idea to the setting of the GLSM and to revisit its analysis to avoid the use of the corresponding far field operator. To do so we need to introduce the following operator, which is closely connected to $S$ and a technical lemma.

$$
S_{\partial \Omega}: H^{-\frac{1}{2}}(\partial \Omega) \rightarrow H^{\frac{1}{2}}(\partial \Omega), S_{\partial \Omega}(f)(x)=\int_{\partial \Omega} \Phi(x, y) f(y) d s(y), x \in \partial \Omega
$$

Lemma 12. If $k^{2}$ is not a Dirichlet eigenvalue of the Laplace operator in $\Omega$, we have that:

$$
S_{\partial \Omega}^{*} S_{\partial \Omega}^{-1} \bar{S}^{*}=S^{*}
$$

Proof. If $k^{2}$ is not a Dirichlet eigenvalue of the Laplace operator in $\Omega$, for $\varphi \in H^{-\frac{1}{2}}(\partial \Omega)$ we have that $\bar{S} S_{\partial \Omega}^{-1, *} S_{\partial \Omega} \varphi$ and $S \varphi$ solves the Helmholtz equation in $\Omega$. Straightforward calculations provide that $\bar{S}_{\partial \Omega} S_{\partial \Omega}^{-1, *} S_{\partial \Omega}^{*} \varphi=\bar{S}_{\partial \Omega} \varphi$ therefore the two solutions share the same boundary values on $\partial \Omega$. By taking the adjoint we conclude the proof.

Using (42) we arrived at

$$
B=S_{\partial \Omega}^{*} S_{\partial \Omega}^{-1} N=S^{*} T S
$$

From this factorization one can either use the framework developed in [3] or the factorization method developed in [13]. One can also apply the results from Section 3.1 by substituting $\langle F g-\phi, g\rangle$ with $\langle C(F g-\phi), g\rangle$.

5.2. Point sources and measurements lying on different surfaces. One can consider a limited aperture nearfield measurement by considering that the point sources are located on $\Gamma_{s} \subset \partial \Omega$ and the measurements are done on $\Gamma_{m} \subset \partial \Omega$ and assume that $\Gamma_{s}$ and $\Gamma_{m}$ are analytic surfaces. In this case similarly to the far field case we obtain a factorization :

$$
N=\bar{S}_{m}^{*} T S_{s},
$$

where $S_{m}$ and $S_{s}$ are defined similarly to $S_{\partial \Omega}$ with $\partial \Omega$ replaced by $\Gamma_{m}$ and $\Gamma_{s}$ respectively. Similarly to Section 2, we define the compact operator $G$ from $\overline{\mathcal{R}\left(S_{s}\right)}$ to $H^{\frac{1}{2}}\left(\Gamma_{m}\right)$ by $G:=\bar{S}_{m}^{*} T$. As for the far field case we have the following result which is proven in [13],

Lemma 13. If Hypothesis 1 is verified, $\Phi_{z} \in \mathcal{R}(G)$ if and only if $z \in D$.

Lemma 14. If $k$ is not a dirichlet eigenvalue of $\Omega$ we have that $S_{s}$ and $S_{m}$ are dense in $\left\{v \in L^{2}(D)\right.$ s.t. $\Delta v+v=0$ in $\left.D\right\}$ 
As already pointed out the operator $T$ is not changed by the type of incident wave and measurement therefore it keeps the coercivity property given in section 4 .

The two previous lemmas, the coercivity of $T$ and (44) are all the required ingredients to apply the framework of section 3.2 with $V=S_{s}, U=\bar{S}_{m}$ and $F=N$. We therefore obtain the following corollary for the GLSM with nearfield measurements.

Corollary 15. Assume that Hypotheses 1 and 2 hold and that $D \subset \Sigma$. Then $z \in D$ if and only if

$\left|\left\langle N g_{2}^{z, \alpha}, g_{1}^{z, \alpha}\right\rangle\right|+\alpha^{-\eta}\left\|S_{s} g_{2}^{z, \alpha}-\bar{S}_{m} g_{1}^{z, \alpha}\right\|_{L^{2}(\Sigma)}^{2}$ remains bounded for $g_{1}^{z, \alpha}$ and $g_{2}^{z, \alpha}$ defined as in Section 3 with $\phi=\phi_{z}$

$R\left(g^{z, \alpha, \delta}, \alpha, \delta\right)$ (defined in Theorem 6) remains bounded for $g^{z, \alpha, \delta}$ defined as in Section 3 with $\phi=\phi_{z}, V=S_{s}$ and $U=\bar{S}_{m}$.

Moreover we have that one can extract a subsequence from the sequence of herglotz wave functions associated to $g^{z, \alpha}$ (resp. $g^{z, \alpha, \delta}$ ) which will converge strongly to the solution $v$ of (38) with $(f, g)=\left(\Phi_{z}, \frac{\partial \Phi_{z}}{\partial \nu}\right)$ as $\alpha$ goes to zero (resp. as $\alpha$ and $\delta$ go to zero for $\left.\delta \leq \delta_{0}\right)$.

6. Numerical Algorithm and results. In order to fix the ideas, we shall restrict ourselves in a two dimensional setting with far field measurement. We identify $\mathbb{S}^{1}$ with the interval $[0,2 \pi[$. In order to collect the data of the inverse problem we solve numerically (1) for $\mathrm{N}$ incident fields using the surface integral equation forward solver available in [11]. The discrete version of $F$ is then the matrix $F_{\mathrm{N}}$. We add some noise to the data to build a noisy far field matrix $F_{N}^{\delta}$ where $\left(F_{\mathrm{N}}^{\delta}\right)_{j, k}=\left(F_{\mathrm{N}}\right)_{j, k}\left(1+\sigma N_{i j}\right)$ for $\sigma>0$ and $N_{i j}$ an uniform complex random variable in $[-1,1]^{2}$. We denote $\Phi_{z, \mathrm{~N}} \in \mathbb{C}^{\mathrm{N}}$, the vector defined by $\Phi_{z, \mathrm{~N}}(j)=\phi_{z}\left(\frac{2 \pi j}{\mathrm{~N}}\right)$ for $0 \leq j \leq \mathrm{N}-1$. In all our experiments we take $\eta=0$ as we do not find a significant influence for this parameter.

6.1. Symmetric case. First we will look at the result given when $\Gamma_{m}=\Gamma_{s}$. This setting could be seen as a reference image as it does not introduce any new regularization term based on a priori knowledge on $D$ (the choice of $\Sigma$ ). Moreover it can be formulated [3] as a convex functional if one introduces $F_{\#}^{\delta}=\left|\Re\left(F^{\delta}\right)\right|+\left|\Im\left(F^{\delta}\right)\right|$, we introduce:

$$
g_{\#}^{z, \alpha, \delta}=\arg \min _{g \in \mathbb{C}^{N}} \alpha\left\|\left(F_{\#}^{\delta}\right)^{\frac{1}{2}} g\right\|^{2}+\alpha^{1-\eta} \delta\|g\|^{2}+\left\|F^{\delta} g-\phi_{z}\right\|^{2}
$$

This minimization is solved using the normal equation:

$$
g_{\#}^{z, \alpha, \delta}=\left(\alpha F_{\#}+\alpha^{1-\eta} \delta I d+F^{\delta, *} F^{\delta}\right)^{-1} F^{\delta, *} \phi_{z}
$$

And finally we use the following indicator function to retrieve the $D$

$$
\mathcal{I}_{\#}(z)=\frac{1}{\left\|\left(F_{\#}^{\delta}\right)^{\frac{1}{2}} g_{\#}^{z, \alpha, \delta}\right\|^{2}+\alpha^{-\eta} \delta\left\|g_{\#}^{z, \alpha, \delta}\right\|^{2}}
$$

To compare with setting where $\Gamma_{m} \neq \Gamma_{s}$ we also introduced :

$$
g^{z, \alpha, \delta}=\arg \min _{g \in \mathbb{C}^{N}} \alpha\left|\left\langle F^{\delta} g, g\right\rangle\right|+\alpha^{1-\eta} \delta\|g\|^{2}+\alpha^{1-\eta}\left|\left\langle F^{\delta} g-\phi_{z}, g\right\rangle\right|+\left\|F^{\delta} g-\phi_{z}\right\|^{2}
$$


and consider the following indicator function:

$$
\mathcal{I}(z)=\frac{1}{\left|\left\langle F^{\delta} g^{z, \alpha, \delta}, g^{z, \alpha, \delta}\right\rangle\right|+\alpha^{-\eta} \delta\left\|g^{z, \alpha, \delta}\right\|^{2}} .
$$

Computing $g^{z, \alpha, \delta}$ is much more challenging as the functional is non convex nor differentiable in general. In [3], a first order gradient method is used. We here improve the efficiency of this scheme by using a second order method. We give the formula of the gradient and the hessian explicitly in the more general case were $\Gamma_{m} \neq \Gamma_{s}$. The iteration are initialized by using the original LSM [4] with Tikhonov regularization :

$$
g_{0}^{z, \beta, \delta}=\arg \min _{g \in \mathbb{C}^{N}} \beta\|g\|^{2}+\left\|F^{\delta} g-\phi_{z}\right\|^{2}
$$

where we choose $\beta$ such that $\delta\left\|g_{0}^{z, \beta, \delta}\right\|=\left\|F_{0}^{z, \beta, \delta}-\phi_{z}\right\|$. From this choice of $\beta$ we set $\alpha=\frac{\beta}{\left\|F_{\#}\right\|}$ or $\frac{\beta}{\|F\|}$.

We consider two examples one with two ellipses and one with a kite shape obstacle both penetrable obstacle with index of refraction of 0.2 . The axis are labelled as multiple of the wavelength $\lambda=2 \pi / k$. We consider three apertures : $[\pi / 2,3 \pi / 2[,[3 \pi / 4,5 \pi / 4[$ and $[7 \pi / 8,9 \pi / 8[$ with a noise $\delta=1 \%$. In figure 2 , we show the results of $\mathcal{I}_{\#}$ and $\mathcal{I}$.

6.2. NonSymetric case. We consider the case where $\Gamma_{m} \neq \Gamma_{s}$. In this case we have to define $g^{z, \alpha, \delta}$ as the minimizer of a (non convex nor differentiable) cost functional,

$$
\begin{aligned}
g^{z, \alpha, \delta}=\arg \min _{g \in \mathbb{C}^{N} \times \mathbb{C}^{N}} & \alpha\left|\left\langle F^{\delta} g_{2}, g_{1}\right\rangle\right|+\alpha^{1-\eta} \delta\|g\|^{2}+\alpha^{1-\eta}\left|\left\langle F^{\delta} g_{2}-\phi_{z}, g_{1}\right\rangle\right| \\
& +\alpha^{1-\eta}\left\|H_{s} g_{2}-H_{m} g_{1}\right\|^{2}+\left\|F^{\delta} g_{2}-\phi_{z}\right\|^{2}
\end{aligned}
$$

and we introduced the indicator function:

$$
\mathcal{I}(z)=\frac{1}{\left|\left\langle F^{\delta} g_{2}^{z, \alpha, \delta}, g_{1}^{z, \alpha, \delta}\right\rangle\right|+\alpha^{-\eta} \delta\left\|g^{z, \alpha, \delta}\right\|^{2}+\alpha^{-\eta}\left\|H_{s} g_{2}^{z, \alpha, \delta}-H_{m} g_{1}^{z, \alpha, \delta}\right\|^{2}}
$$

To minimize the cost functional we will rely on a second order descent method. We will choose the starting point of the descent, $g_{0}$, as

$$
\begin{gathered}
g_{0,2}^{z, \beta_{2}, \delta}=\arg \min _{g \in \mathbb{C}^{N}} \beta_{2}\|g\|^{2}+\left\|F^{\delta} g-\phi_{z}\right\|^{2} \\
g_{0,1}^{z, \beta_{1}, \delta}=\arg \min _{g \in \mathbb{C}^{N}} \beta_{1}\|g\|^{2}+\left\|H_{m} g-H_{s} g_{0,2}^{z, \beta, \delta}\right\|^{2}
\end{gathered}
$$

where we choose $\beta_{2}$ such that $\delta\left\|g_{0,2}^{z, \beta_{2}, \delta}\right\|=\left\|F_{0,2}^{z, \beta_{2}, \delta}-\phi_{z}\right\|$ and $\beta_{1}$ such that $\left\|g_{0,1}^{z, \beta_{1}, \delta}\right\|=$ $\left\|g_{0,2}^{z, \beta_{2}, \delta}\right\|$. This second choice is purely arbitrary, our purpose in setting $\beta_{1}$ is to avoid, $g_{0,1}^{z, \beta_{1}, \delta}$ to have large norm which would dominate numerically all other quantities. 
The minimization of $J_{\alpha}^{\delta}$ causes numerical problem. Indeed first numerically $H_{m}$ is a compact operator and even if it is not important for the theory we are implicitly inverting it by minimizing $J_{\alpha}^{\delta}$ therefore we have to be careful on the balance between the terms $\left\|g_{1}\right\|^{2}$ and $\left\|H_{m} g_{1}-H_{s} g_{2}\right\|$. This is even more important as $H_{s} g_{2}$ is not in the range of $H_{m}$. Since the theory does not give a strategy to set $\alpha$, we proposed and tested three strategies that give similar results. Those strategies are based on the idea (we also use to pick an initial guess $g_{0}$ ) that $\left\|g_{1}^{z, \beta_{1}, \delta}\right\|$ and $\left\|g_{2}^{z, \beta_{1}, \delta}\right\|$ should have the same order of magnitude.

First one should remark that we have used the same parameter, $\eta$ in front of all the terms but it could have been chosen with a different value for each term (as long as it stays between 0 and 1 for the theory). Using different $\alpha$ instead of $\eta$ to keep simple notation we introduce:

$$
\begin{aligned}
J_{\alpha}^{\delta}\left(g_{1}, g_{2}\right)= & \alpha\left|\left\langle F^{\delta} g_{2}, g_{1}\right\rangle\right|+\alpha_{1} \delta\left\|g_{1}\right\|^{2}+\alpha_{2} \delta\left\|g_{2}\right\|^{2}+\alpha^{1-\eta}\left|\left\langle F^{\delta} g_{2}-\phi_{z}, g_{1}\right\rangle\right| \\
& +\alpha_{3}\left\|H_{s} g_{2}-H_{m} g_{1}\right\|^{2}+\left\|F^{\delta} g_{2}-\phi_{z}\right\|^{2}
\end{aligned}
$$

We have actually increase the number of parameter in order to get some freedom to balance the term involving $g_{1}$ and $H_{m} g_{1}$. To set $\alpha$ we use again our heuristic: $\alpha=\frac{\beta_{2}}{\|F\|}$. We propose to choose $\alpha_{1}=\alpha_{2}=\alpha$ and $\alpha_{3}=\alpha \delta / \beta_{1}$ and therefore keep the regularizing power used to find the initial guess. The parameters set, we used a newton method to minimize $J_{\alpha}^{\delta}$.

A second solution we have experienced is to alternatively minimize $J_{\alpha}^{\delta}$ as a function of $g_{2}$ with $\alpha_{3}=\alpha_{1}=0$ and to minimize the same Tikhonov functional (45) we used to find the initial guess $g_{0,1}$. This will impose $\left\|g_{1}^{z, \beta_{1}, \delta}\right\|=\left\|g_{2}^{z, \beta_{2}, \delta}\right\|$ and limit the number of parameters to set, however it is not a scheme that is cover by the theory.

A third solution closely related to our heuristic for symmetric factorization, we have set $\alpha_{3}$ to 1 and $\alpha_{1}=\alpha_{2}=\alpha$ where $\alpha$ is chosen to be equal to $\max \left(\beta_{1}, \beta_{2}\right) /\left\|F^{\delta}\right\|$.

All those three methods give similar result. In the following we will show only the results of the first method. In order to perform the Newton method we need to compute the gradient and the Hessian which we explicit in the following for the original cost functional, both gradient and Hessian can be easily derive from those formulas. If $\cdot$ is the dot product without conjugate, ${ }^{t}$ the transposition and by ${ }^{*}$ the classical transpose-conjugate, we can rewrite $J_{\alpha}^{\delta}(\phi, \cdot)$ :

$\alpha\left|g^{*} \cdot\left(F^{\delta} g\right)\right|+\alpha^{1-\eta}\left|g^{*} \cdot\left(F^{\delta} g-\phi\right)\right|+\delta \alpha^{1-\eta}\left\|F^{\delta}\right\| g^{*} \cdot g+\alpha^{1-\eta}(H g)^{*} \cdot(H g)+\left(F^{\delta} g-\phi\right)^{*} \cdot\left(F^{\delta} g-\phi\right)$

where we use the matrix:

$$
F^{\delta}=\left[\begin{array}{cc}
0 & F_{\mathrm{N}}^{\delta} \\
0 & 0
\end{array}\right] \text { and } H=\left[\begin{array}{ll}
H_{m} & -H_{s}
\end{array}\right] \text { and } g=\left[\begin{array}{c}
g_{2} \\
g_{1}
\end{array}\right] \text { and } \phi=\left[\begin{array}{c}
\Phi_{z} \\
0
\end{array}\right]
$$

Using this notation we can compute following the framework of [14] the gradient

$$
\begin{aligned}
\delta \alpha^{1-\eta}\left\|F^{\delta}\right\| g+F^{\delta *}\left(F^{\delta} g-\phi\right)+\alpha^{1-\eta} H^{*} H g & +\alpha \frac{\overline{g^{*} \cdot\left(F^{\delta} g\right)} F^{\delta} g+\left(g^{*} \cdot\left(F^{\delta} g\right)\right) F^{\delta *} g}{|| g^{*} \cdot\left(F^{\delta} g\right) \mid} \\
& +\alpha^{1-\eta} \frac{\frac{\left(g^{*} \cdot\left(F^{\delta} g-\phi\right)\right)\left(F^{\delta} g-\phi\right)+\left(g^{*} \cdot\left(F^{\delta} g-\phi\right)\right) F^{\delta *} g}{\left|g^{*} \cdot\left(F^{\delta} g-\phi\right)\right|}}{}
\end{aligned}
$$


and the Hessian,

$$
\begin{aligned}
& \delta \alpha^{1-\eta}\left\|F^{\delta}\right\| I d+F^{\delta *} F^{\delta}+\alpha^{1-\eta} H^{*} H+\alpha \frac{\overline{g^{*} \cdot\left(F^{\delta} g\right)} F^{\delta}+\left(g^{*} \cdot\left(F^{\delta} g\right)\right) F^{\delta *}+F^{\delta} g g^{*} F^{\delta *}+F^{\delta *} g g^{*} F^{\delta}}{\left|g^{*} \cdot\left(F^{\delta} g\right)\right|} \\
& -\alpha \frac{\left(\overline{g^{*} \cdot\left(F^{\delta} g\right)} F^{\delta} g+\left(g^{*} \cdot\left(F^{\delta} g\right)\right) F^{\delta *} g\right)\left(\overline{g^{*} \cdot\left(F^{\delta} g\right)} F^{\delta} g+\left(g^{*} \cdot\left(F^{\delta} g\right)\right) F^{\delta *} g\right)^{*}}{2\left|g^{*} \cdot\left(F^{\delta} g\right)\right|^{\frac{3}{2}}} \\
& +\alpha^{1-\eta}\left(\overline{\left(\frac{\left(g^{*} \cdot\left(F^{\delta} g-\phi\right)\right)}{\delta} F^{\delta}+\left(g^{*} \cdot\left(F^{\delta} g-\phi\right)\right) F^{\delta *}+\left(F^{\delta} g-\phi\right)\left(g^{*} F^{\delta *}-\phi^{*}\right)+F^{\delta *} g g^{*} F^{\delta}\right.}\right. \\
& -\frac{\left(\overline{\left(g^{*} \cdot\left(F^{\delta} g-\phi\right)\right)}\left(F^{\delta} g-\phi\right) \mid\right.}{2\left|F^{*} \cdot\left(F^{\delta} g-\phi\right)\right|^{\frac{3}{2}}}
\end{aligned}
$$

We apply those techniques to the case of back scattering data which is when $\Gamma_{m}=-\Gamma_{s}$, for apertures $\Gamma_{s}=[\pi / 2,3 \pi / 2[,[3 \pi / 4,5 \pi / 4[$ and $[7 \pi / 8,9 \pi / 8[$. The result are shown in figure 3 for the kite example for a domain $\Sigma$ which occupies the whole image and the smallest rectangle that contains $D$. We also consider the case of $\Gamma_{s}$ being either $[\pi / 2,3 \pi / 2[,[3 \pi / 4,5 \pi / 4[$ and $\left[7 \pi / 8,9 \pi / 8\left[\right.\right.$ and $\Gamma_{m}$ being either $[0, \pi[,[\pi / 4,3 \pi / 4[$ and $[3 \pi / 8,5 \pi / 8[$. The results are shown in figure 4, again for a kite example and an original setting of sources and measurements. On those simulation the size of $\Sigma$ has no clear impact therefore we will only show simulation for the large grid.

Figures 5 and 6 consider backscattering data from aperture of the same size as previously, but rotated around the obstacle. We see the strong dependency with the mean direction of the aperture. The fact that the results are coherent with the aperture we consider lets us think that non symmetric aperture is intrinsically worst than symmetric one. Connected to that subject in [9] they study invisibility for a finite number of incident direction and demonstrate that imposing invisibility in symmetric direction is equivalent to impose invisibility in all direction. Meaning that there is more information inside symmetric-factorization like far field operator than any other setting of sources and measurements.

\section{REFERENCES}

[1] Lorenzo Audibert. Sampling method for sign changing contrast. 2016. Preprint.

[2] Lorenzo Audibert, Alexandre Girard, and Houssem Haddar. Identifying defects in an unknown background using differential measurements. Inverse Problems and Imaging, 9(3):625-643, 2015.

[3] Lorenzo Audibert and Houssem Haddar. A generalized formulation of the linear sampling method with exact characterization of targets in terms of farfield measurements. Inverse Problems, 30(3):035011, 2014.

[4] Fioralba Cakoni and David Colton. Qualitative methods in inverse scattering theory. Interaction of Mechanics and Mathematics. Springer-Verlag, Berlin, 2006. An introduction.

[5] Fioralba Cakoni and Isaac Harris. The factorization method for a defective region in an anisotropic material. Inverse Problems, 31(2):025002, 22, 2015.

[6] David Colton, Houssem Haddar, and Michele Piana. The linear sampling method in inverse electromagnetic scattering theory. Inverse Problems, 19(6):S105-S137, 2003. Special section on imaging.

[7] David Colton and Andreas Kirsch. A simple method for solving inverse scattering problems in the resonance region. Inverse Problems, 12(4):383-393, 1996.

[8] David Colton and Rainer Kress. Inverse acoustic and electromagnetic scattering theory, volume 93 of Applied Mathematical Sciences. Springer, New York, third edition, 2013.

[9] Anne-Sophie Bonnet-Ben Dhia, Lucas Chesnel, and Sergei A Nazarov. Non-scattering wavenumbers and far field invisibility for a finite set of incident/scattering directions. Inverse Problems, 31(4):045006, 2015.

[10] Y. Grisel, V. Mouysset, P.-A. Mazet, and J.-P. Raymond. Determining the shape of defects in non- 
absorbing inhomogeneous media from far-field measurements. Inverse Problems, 28(5):055003, 19, 2012.

[11] H. Haddar. Sampling 2d, Mars 2013. http://sourceforge.net/projects/samplings-2d/.

[12] Guanghui Hu, Jiaqing Yang, Bo Zhang, and Haiwen Zhang. Near-field imaging of scattering obstacles with the factorization method. Inverse Problems, 30(9):095005, 25, 2014.

[13] Andreas Kirsch and Natalia Grinberg. The factorization method for inverse problems, volume 36 of Oxford Lecture Series in Mathematics and its Applications. Oxford University Press, Oxford, 2008.

[14] L. Sorber, M. Barel, and L. Lathauwer. Unconstrained optimization of real functions in complex variables. SIAM J. Optim., 22(3):879-898, 2012.

[15] John Sylvester. Discreteness of transmission eigenvalues via upper triangular compact operators. SIAM J. Math. Anal., 44(1):341-354, 2012. 

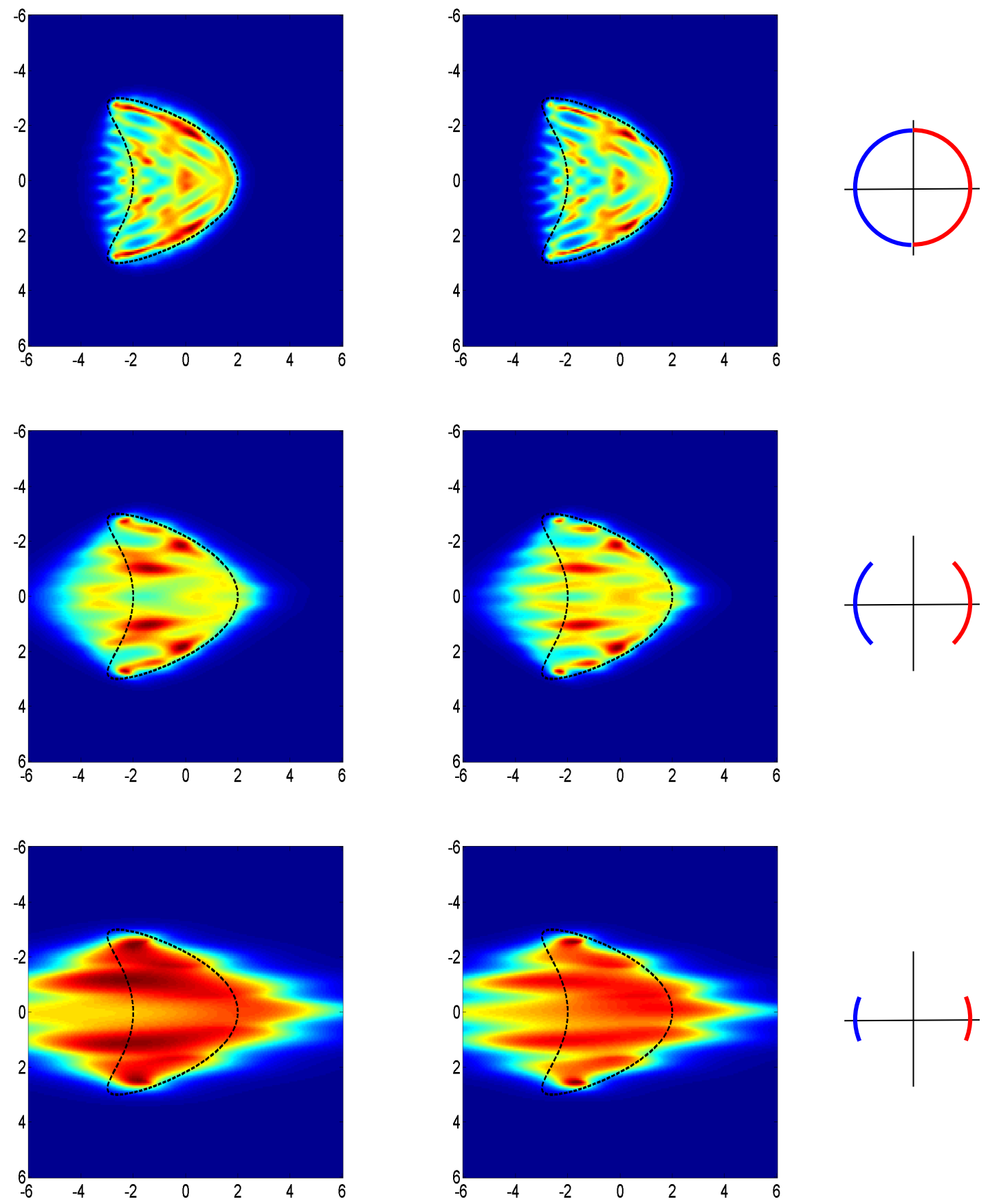

Figure 2. On the left $\mathcal{I}_{\#}$ and on the right $\mathcal{I}$. From up to down the aperture is : $[\pi / 2,3 \pi / 2[,[3 \pi / 4,5 \pi / 4[$ and $[7 \pi / 8,9 \pi / 8[$ (as depicted on the right column). 

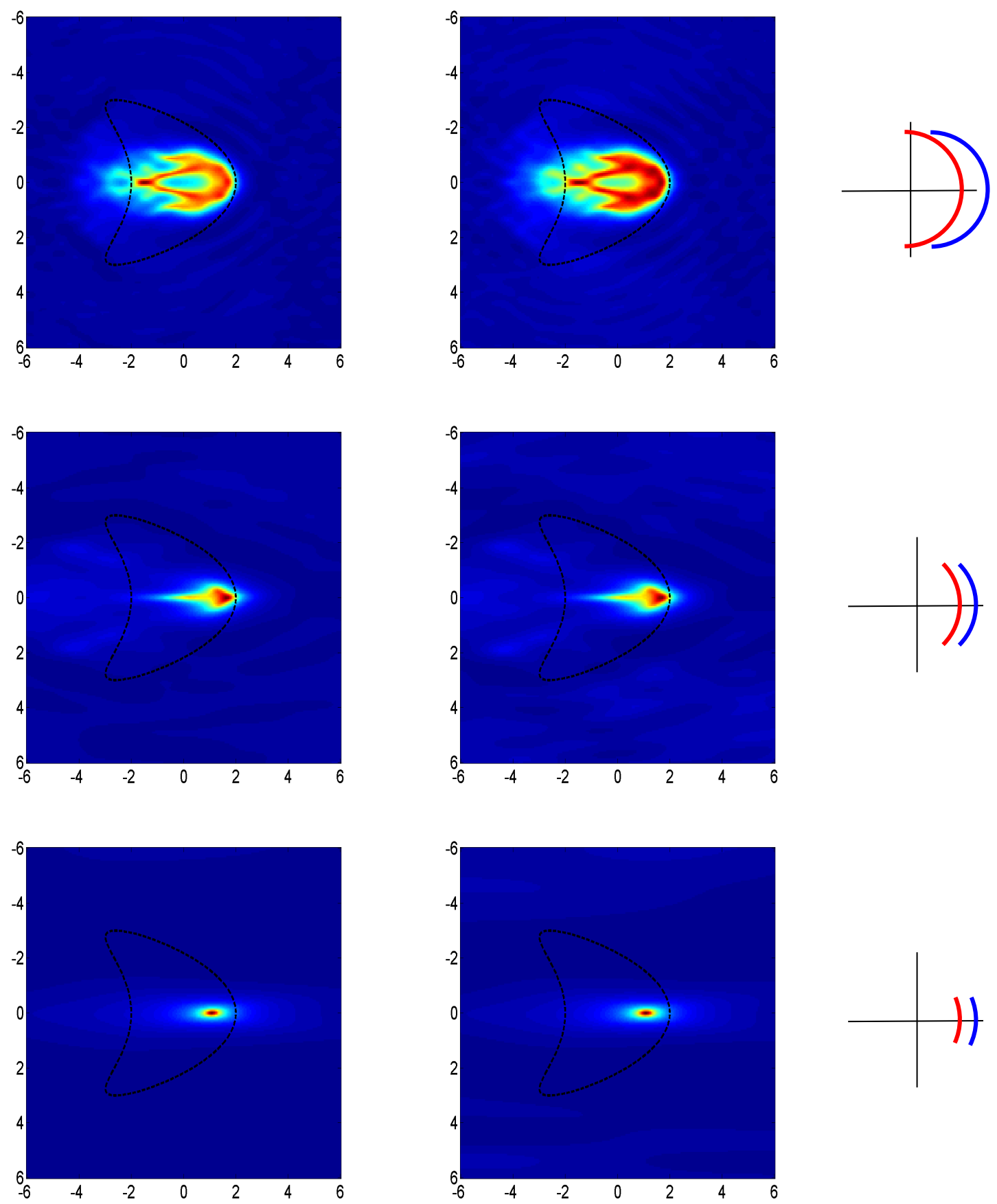

Figure 3. $\mathcal{I}$ computed on the left with a large $\Sigma$ and with on the right with a small one. From up to down the apertures are : $\Gamma_{s}=[\pi / 2,3 \pi / 2[,[3 \pi / 4,5 \pi / 4[$ and $[7 \pi / 8,9 \pi / 8[$ (as depicted in the right column). 

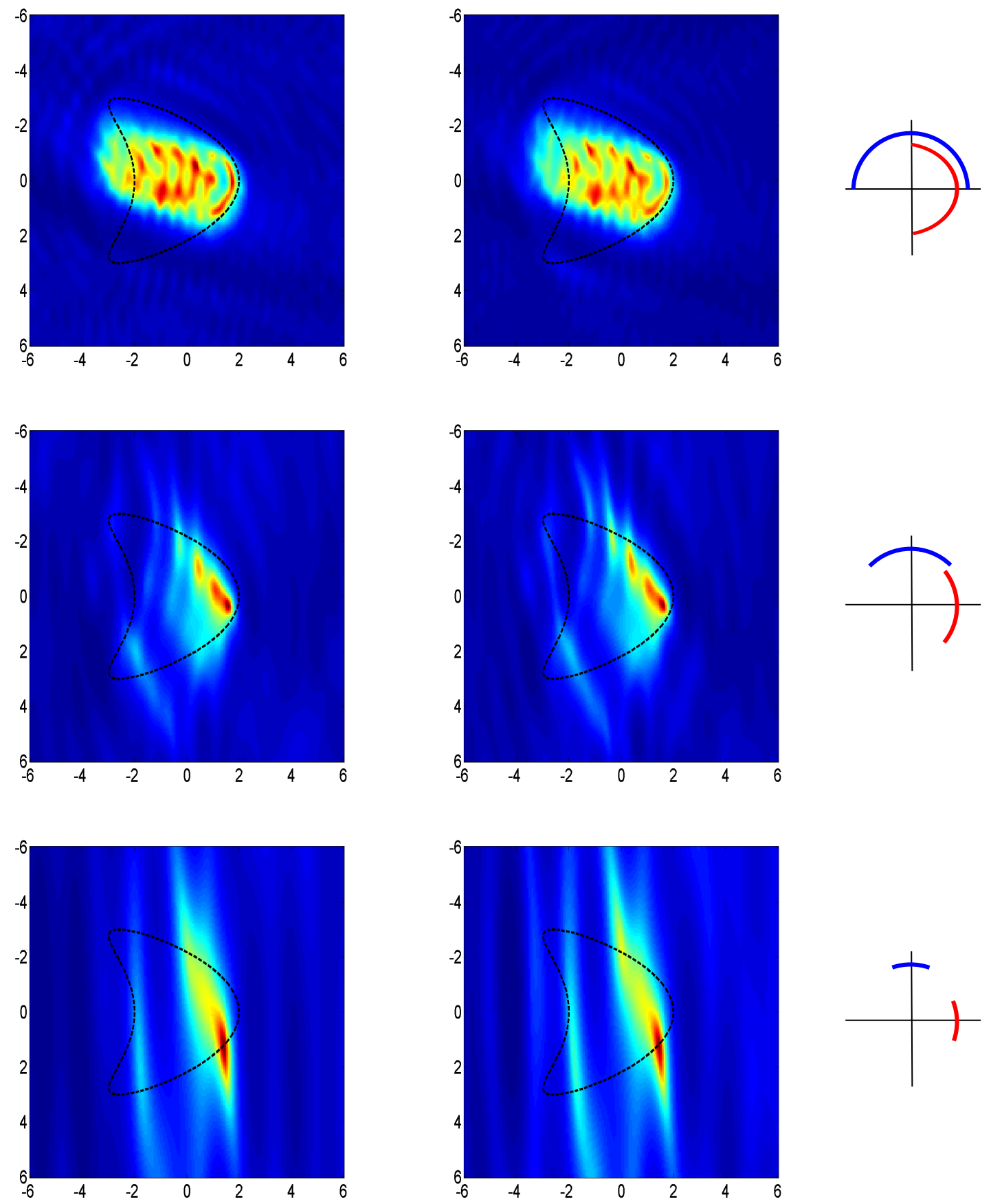

Figure 4. $\mathcal{I}$ computed on the left with a large $\Sigma$ and with on the right with a small one. From up to down the apertures are $\Gamma_{s}=\left[\pi / 2,3 \pi / 2\left[,\left[3 \pi / 4,5 \pi / 4\left[\right.\right.\right.\right.$ and $\left[7 \pi / 8,9 \pi / 8\left[\right.\right.$ and $\Gamma_{m}=[0, \pi[,[\pi / 4,3 \pi / 4[$ and $[3 \pi / 8,5 \pi / 8[($ as depicted in the right column). 

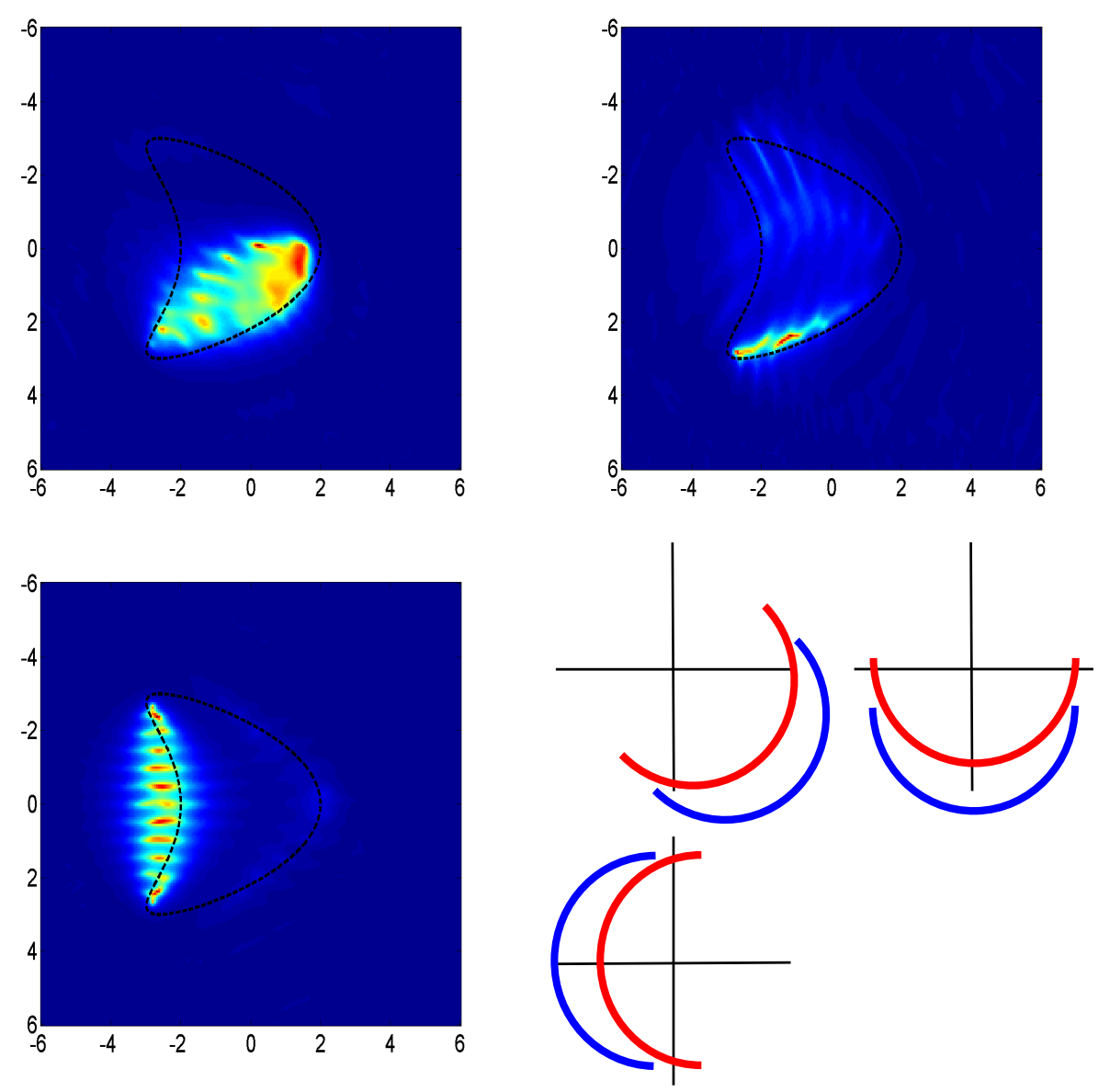

Figure 5. $\mathcal{I}$ computed with $\Sigma$ equals the full grid. From left to right and up to down the aperture are : $\Gamma_{s}\left[3 \pi / 4,7 \pi / 4\left[,\left[\pi, 2 \pi\left[\right.\right.\right.\right.$ and $\left[-\pi / 2, \pi / 2\left[\right.\right.$ and $\Gamma_{m}=\Gamma_{s}+\pi$ (the sensor setting are depicted following the same order in the last image). 

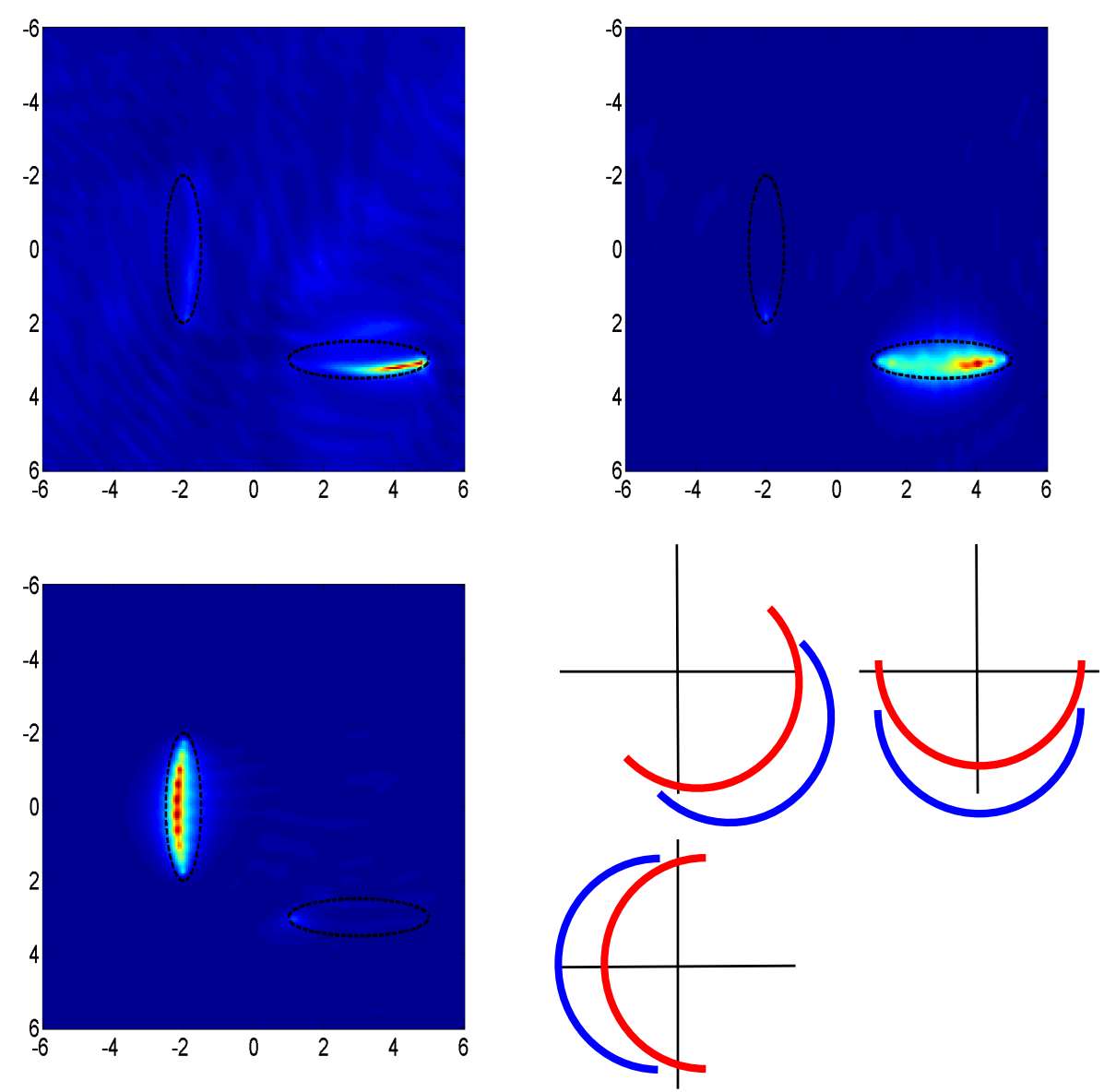

Figure 6. $\mathcal{I}$ computed with $\Sigma$ equals the full grid. From left to right and up to down the aperture are : $\Gamma_{s}=\left[3 \pi / 4,7 \pi / 4\left[,\left[\pi, 2 \pi\left[\right.\right.\right.\right.$ and $\left[-\pi / 2, \pi / 2\left[\right.\right.$ and $\Gamma_{m}=\Gamma_{s}+\pi$ (the sensor setting are depicted following the same order in the last image). 